\title{
Intermediate length scale dynamics of polyisobutylene
}

\author{
B. Farago, ${ }^{1}$ A. Arbe, ${ }^{2}$ J. Colmenero, $, 2,3,4$ R. Faust,${ }^{5}$ U. Buchenau, ${ }^{6}$ and D. Richter ${ }^{6}$ \\ ${ }^{1}$ Institut Laue-Langevin, 156X, 38042 Grenoble Cedex, France \\ ${ }^{2}$ Unidad de Física de Materiales (CSIC-UPV/EHU), Apartado 1072, 20080 San Sebastián, Spain \\ ${ }^{3}$ Departamento de Física de Materiales, Universidad del País Vasco UPV/EHU, Apartado 1072, 20080 San Sebastián, Spain \\ ${ }^{4}$ Donostia International Physics Center, Apartado 1072, 20080 San Sebastián, Spain \\ ${ }^{5}$ Polymer Science Program, Department of Chemistry, University of Massachusetts-Lowell, One University Avenue, Lowell, \\ Massachusetts 01854 \\ ${ }^{6}$ Institute für Festkörperforschung, Forschungszentrum Jülich, 52425 Jülich, Germany
}

(Received 12 December 2001; published 21 May 2002)

\begin{abstract}
We report on a neutron spin echo investigation of the intermediate scale dynamics of polyisobutylene studying both the self-motion and the collective motion. The momentum transfer $(Q)$ dependences of the self-correlation times are found to follow a $Q^{-2 / \beta}$ law in agreement with the picture of Gaussian dynamics. In the full $Q$ range of observation, their temperature dependence is weaker than the rheological shift factor. The same is true for the stress relaxation time as seen in sound wave absorption. The collective times show both temperature dependences; at the structure factor peak, they follow the temperature dependence of the viscosity, but below the peak, one finds the stress relaxation behavior.
\end{abstract}

DOI: 10.1103/PhysRevE.65.051803

PACS number(s): 61.41.+e, 61.12.Ex, 64.70.Pf

\section{INTRODUCTION}

Experimental results on the collective relaxational motions at intermediate length scales are a white area on the landscape of the dynamics of glass-forming liquids and even more so of polymers. Intermediate scales denote the regime of lengths larger than the interparticle distances but smaller than the hydrodynamic range. The reasons for this deficiency are (i) the very low scattered intensity in the range of momentum transfers $Q=4 \pi \sin (\theta / 2) / \lambda$ ( $\lambda$ is the wavelength of radiation; $\theta$ is the scattering angle) below the first maximum of the static structure factor; (ii) the important role of multiple scattering which originates from the primary scattering at the first structure factor peak; and (iii) the slow time scales of the motions demanding high-resolution methods to be employed.

From a theoretical point of view the dynamics of glassforming polymers in the intermediate length scale region are also far from understood. During the last years, the mode coupling theory (MCT) $[1,2]$ has been widely used for describing the dynamical features in some glass-forming systems in the neighborhood of the first maximum of the static structure factor. Whether this approach applies also at length scales in the intermediate range order, and in particular for polymers, is still an open question.

At intermediate length scales the macromolecular character of polymers reveals itself. There the large-scale motions are largely determined by chain connectivity effects or entropy. Theories are available for single chain dynamics. Where topological interactions are not yet important, the Rouse model [3] has been particularly successful for describing polymer melts dynamics. At shorter length scales where the simplifying assumptions of the Rouse model do not hold, the influence of the local chain structure becomes important. The approach proposed by Allegra and Ganazzoli $[4,5]$ includes local potentials and gives experimental results in a number of polymers [4-6]. However, the collective motion of an ensemble of different chains has not been calculated so far.

The relation between collective motion and self-motion in simple monatomic liquids was theoretically deduced by de Gennes [7] applying the second sum rule to a simple diffusive process. Phenomenological approaches such as those proposed by Vineyard [8] and Sköld [9] generalize the de Gennes narrowing. The way these theories develop towards the hydrodynamic range is not yet determined to our knowledge.

New theoretical interest in this intermediate length scale regime arose (i) from the outcome of various recent computer simulations that indicated strong collectivity of the motion; in particular, the one-dimensional stringlike cooperative motion of a number of particles, first seen in the relaxation of the glass phase [10], was identified as the dominating mechanism for the relaxational dynamics in simple models of undercooled liquids and as the source of the dynamic heterogeneities in such systems [11,12]; (ii) from the observation of the so-called mesoscopic dynamics in glass-forming materials that are classified as boson peak and fast process [13], and (iii) from the concept of the elusive cooperative volume related to the glass transition [14].

Recently with the advent of high-resolution x-ray techniques the investigation of the collective fluctuations outside the hydrodynamic limit have experienced a rebirth and a significant number of studies on Brillouin scattering in this intermediate length scale regime has been published [15]. In contrast the relaxational regime around energy transfer zero has basically not yet been tackled. While quasielastic light scattering is the prominent technique to explore the hydrodynamic regime at length scales in the order of several 100 $\mathrm{nm}$, the intermediate scale regime at present can only be accessed by neutrons.

In this paper we present such a study on polyisobutylene (PIB), a polymer that is well investigated by a whole range of techniques. The viscoelastic properties are among those of 
the most thoroughly investigated properties of any polymer [16-24]. The full ensemble of spectroscopic techniques ranging from dielectric spectroscopy [25-27], nuclear magnetic resonance (NMR) [28,29], to spin label techniques [21] has been applied. Also a number of high-resolution neutron scattering studies are available $[6,25,30]$ providing important background information for the present experiments. The key results are as listed below.

(i) Below $10 \mathrm{MHz}$, a consistent time-temperature superposition of different kinds of mechanical data in terms of the William-Landel-Ferry (WLF) equation [20] is achieved. However, a high-frequency Brillouin scattering experiment at high temperature $(473 \mathrm{~K})$ does not seem to fit into this picture [23].

(ii) The structural relaxation, manifested by the relaxation of the structure factor at its first maximum, has been found to follow with high precision the viscosity temperature shift factor [25].

(iii) The secondary relaxation has been investigated in a combined effort of dielectric spectroscopy and quasielastic incoherent neutron scattering [30]. These experiments identified the $\beta$ process as a joint motion of the methyl groups and the chain backbone exhibiting a motional amplitude of $2.7 \AA$. Recent molecular dynamics simulations confirm this picture [31].

(iv) Finally, covering a wide $Q$ and time regime, the single chain dynamic structure factor was studied on labeled chains in the melt [6]. Moving from larger to smaller scales translational chain diffusion followed by the entropic Rouse relaxation were identified. The Rouse relaxation was found to be limited towards shorter scales by an intrachain dissipative process first suggested by Allegra and Ganazzoli $[4,5]$.

This work deals with neutron spin echo (NSE) experiments on the self-dynamics and collective dynamics at intermediate length scales of PIB as a function of temperature. Supplementary information was obtained from a study of the static structure factor, in particular in the low- $Q$-regime. Varying the neutron wavelength over a large range we explicitly assessed the effect of multiple scattering and found it to be acceptable for a meaningful study. This was corroborated by a comparison of NSE spectra observed below and above the wavelength of multiple scattering threshold.

Section II is devoted to the theoretical derivation of the scattering from long-range density fluctuations. In Sec. III we describe the sample preparation and characterization and the experimental methods used. In Sec. IV the structure factor results are presented; then we turn to the NSE results on the self-motion before we outlay our measurements on the collective dynamics. Section V places the results into the context of other studies. The outcome is discussed in terms of (i) a low- $Q$ approach that comes from the hydrodynamic limit, (ii) the mode coupling theory and (iii) considerations based on the single polymer chain dynamics. A summary concludes the paper.

\section{THEORETICAL BACKGROUND}

In this section, starting from the second moment sum rule, we derive the scattering function from density fluctuations in the hydrodynamic limit.
The coherent scattering in the low- $Q$ regime is due to long-range density fluctuations. In disordered matter with the atom number density $\rho$ and the isothermal compressibility $\kappa_{T}$, one has in thermodynamic equilibrium at constant pressure,

$$
S(Q \rightarrow 0)=\rho k_{B} T \kappa_{T}=\frac{k_{B} T}{m \nu_{\ell_{o}}^{2}}+\frac{k_{B} T^{2} \alpha^{2}}{m C_{p}} .
$$

In the second part of the equation, the isothermal compressibility is decomposed into the adiabatic compressibility, expressed via the average atomic mass $m$ and the relaxed lowfrequency longitudinal velocity of sound $\nu_{\ell_{0}}$, and the difference between the two compressibilities, given in terms of the cubic thermal expansion coefficient $\alpha$ and the heat capacity per mass unit $C_{p}$. The first term reflects the mechanical part of the density fluctuations, the second the thermal part, corresponding to the two independent variables, pressure and entropy.

The integrated scattering of Eq. (1) includes Brillouin scattering and the central line. With the kinetic constraints for cold neutrons the Brillouin lines are not excited; one sees only the quasielastic central line $S_{\mathrm{qe}}(Q)$. If there is no damping of the Brillouin lines, the Brillouin scattering function is given by

$$
S(Q, \omega)_{\mathrm{Brill}}=\frac{k_{B} T Q^{2}}{2 m \omega^{2}} \delta(\omega \pm \nu \ell Q),
$$

where $\nu_{\ell}$ is the unrelaxed velocity of sound. The subtraction of the Brillouin contribution leads to the thermodynamic prediction

$$
S_{\mathrm{qe}}(Q \rightarrow 0)=\frac{k_{B} T}{m \nu_{\ell_{o}}^{2}}-\frac{k_{B} T}{m \nu_{\ell}^{2}}+\frac{k_{B} T^{2} \alpha^{2}}{m C_{p}} .
$$

Marvin, Aldrich, and Sack have studied ultrasonic attenuation on PIB [24]. At room temperature their measurements reveal sound velocities that corrected for the density of deuterated PIB are $\nu_{\ell_{o}}=1418 \mathrm{~m} / \mathrm{s}$ and $\nu_{l}=2653 \mathrm{~m} / \mathrm{s}$. At room temperature $\alpha=7.7 \times 10^{-4} \mathrm{~K}^{-1}$ and $C_{p}=1680 \mathrm{~J} / \mathrm{kg} \mathrm{K}$ [32]. Inserting these values into Eq. (3) yields $S_{\mathrm{qe}}(Q \rightarrow 0)$ $=0.183$. Note that $80 \%$ of that value comes from the adiabatic compressibility and only $20 \%$ from the entropy term. This is important, because one expects the two contributions to decay with different time constants: the entropy fluctuations occur on a picosecond scale, while the adiabatic compressibility relaxes with the stress relaxation time. Thus the glass-transition time scale should become visible in the relaxation of the adiabatic compressibility.

Commonly polarized quasielastic light scattering experiments (PCS) on glass forming liquids are interpreted in terms of the Mountain theory that heuristically introduces a complex viscosity into the expression for the hydrodynamic modes [33]. The relaxation step incorporated in the complex viscosity transforms itself into a central quasielastic line that via the coupling to the hydrodynamic modes displays the structural relaxation as a $Q$-independent relaxation process at 
low $Q$. Though generally applied, the Mountain concept has an important drawback: it does not fulfill one of the fundamental rules for scattering functions: the second moment sum rule [34],

$$
\int_{-\infty}^{+\infty} \omega^{2} S(Q, \omega) d \omega=\frac{k_{B} T}{m} Q^{2},
$$

which needs to be followed by any scattering function $S(Q, \omega)$. In the following we will derive an expression that properly introduces local relaxation or vibrations into the scattering function for density fluctuations at low $Q$.

If we integrate the contribution of two Brillouin lines [Eq. (2)], we find that they exhaust the second moment sum rule. The existence of a scattering below the Brillouin line requires a weakening of the Brillouin line, a weakening that is attributable to the low-frequency motion at $\omega$.

To calculate the weakening, we follow the usual ansatz of separating the low-frequency atomic motion into extended sound waves and local modes. The local mode may be either a low-frequency vibrational mode, a relaxational jump in a double-well potential or even one of the instantaneous saddle point modes responsible for diffusion. The local mode is described in terms of a normal coordinate $A_{\text {loc }}$, a frequency $\omega_{\text {loc }}$, and a damping $\gamma_{\text {loc }}$. The ratio of the latter two determines whether one deals with a vibrational or a relaxational mode.

At low frequency, the dominating interaction between local mode and phonon is a bilinear coupling with coupling constant $\Lambda_{P}$ to the longitudinal strain $\varepsilon_{11}$ in the $x$ direction (taken to be the direction of propagation of the Brillouin phonon along the momentum transfer of the measurement),

$$
E_{\text {bilinear }}=\Lambda_{P} A_{\text {loc }} \varepsilon_{11} .
$$

The elastic distortion $\varepsilon_{11}$ in the Brillouin phonon with the wave vector $\mathbf{Q}$ is

$$
\varepsilon_{11}=\varepsilon_{0} \cos (\mathbf{Q r}-\Omega t),
$$

where $\Omega=\nu / Q$ is the frequency of the Brillouin phonon at the scattering vector $\mathbf{Q}$ and $\varepsilon_{0}=Q u_{0}$ is the maximum distortion in the longitudinal wave with amplitude $u_{0}$. The bilinear interaction between Brillouin phonon and local mode leads to mode mixing.

As calculated in the Appendix, mode mixing in combination with the validity of the second moment sum rule [Eq. (4)] results in a scattering function for the local process through the coupling to the Brillouin phonons [Eq. (A9)],

$$
S_{\mathrm{loc}}(Q, \omega)=\frac{\Lambda_{P}^{2} k_{B} T}{2 N m^{2} \nu_{\ell}^{4}} F_{\mathrm{loc}}(\omega),
$$

where $N$ denotes the number of atoms and $F_{\text {loc }}(\omega)$ is given by Eq. (A7).

The same bilinear coupling considered so far for the weakening of the Brillouin line at the high frequency $\Omega$ acts as well on the longitudinal phonons at the low frequency $\omega$ of the measurement. There, it is responsible for the damping. Since we deal with a much lower frequency, $\nu /$ is to be replaced by $\nu_{/ \omega}$, the longitudinal sound velocity at the frequency $\omega$. Similarly, $\Lambda_{P}$ attains a smaller value. In order to get the sum rule for $S(Q)$ [Eq. (4)] we need a scaling with the modulus

$$
\Lambda_{P \omega}=\Lambda_{P} \frac{\nu_{\ell \omega}^{2}}{\nu_{\ell}^{2}}
$$

The energy loss of the phonon to the localized mode is given by

$$
\frac{\partial W}{\partial t}=\Lambda_{P \omega} A \frac{\partial \varepsilon_{33}}{\partial t} .
$$

Inserting Eqs. (A1) and (A3) and replacing $\Omega$ by $\omega$, we find

$$
\frac{\partial W}{\partial t}=\Lambda_{P \omega}^{2} \varepsilon_{0}^{2} \frac{\omega\left(\omega_{\mathrm{loc}}^{2}-\omega^{2}\right) \cos \omega t \sin \omega t+\gamma_{\mathrm{loc}} \omega^{2} \sin ^{2} \omega t}{\left(\omega^{2}-\omega_{\mathrm{loc}}^{2}\right)^{2}+\gamma_{\mathrm{loc}}^{2} \omega^{2}} .
$$

The first part of this loss term describes the frequency shift of the sound wave, the second its damping. The time average of this second part does not disappear. This time average is again proportional to the scattering function $S_{\mathrm{loc}}(Q, \omega)$. Since this holds for any local mode, we get a proportionality between the sound wave loss and the low- $Q$ scattering of the local modes. It is conveniently expressed in terms of the loss tangents,

$$
2 \Gamma=\omega \tan \delta=\frac{1}{W} \frac{\partial W}{\partial t},
$$

where $W=N m \nu_{/ \omega}^{2} \varepsilon_{0}^{2}$ is the energy of the sound wave. Thus we obtain

$$
\tan \delta=\frac{\pi \omega \Lambda_{P \omega}^{2} F_{\mathrm{loc}}(\omega)}{2 N m \nu_{\ell \omega}^{2}},
$$

which is in fact proportional to the scattering $S_{\text {loc }}(Q, \omega)$ of the local mode, Eq. (7). Integrating over all local modes, we find the relation between the sound wave loss and the scattering function $S_{1}(\omega)$ below the Brillouin line,

$$
S_{1}(\omega)=\frac{1}{\pi} \frac{k_{B} T}{m \nu_{\ell \omega}^{2}} \frac{\tan \delta}{\omega} .
$$

This is nothing more than the fluctuation-dissipation relation for the low- $Q$ quasielastic scattering from the adiabatic compressibility. In terms of the longitudinal susceptibility $\chi_{11}$ $=1 / C_{11}$,

$$
S_{1}(\omega)=\frac{1}{\pi} \frac{k_{B} T \rho}{m} \frac{\chi_{11}^{\prime \prime}}{\omega}
$$

where $\chi_{11}^{\prime \prime}$ is the imaginary part of the longitudinal susceptibility at the frequency $\omega$. Using the Kramers-Kronig relation for the real part of the susceptibility, one recovers the adiabatic compressibility term of Eq. (1). 
Equation (14) concludes our derivation of the low- $Q$ scattering function connecting local relaxational or vibrational processes with the long-range density fluctuations. The basic ingredients of this derivation were the second sum rule [Eq. (4)] and a mode mixing between sound waves and local vibrator or relaxator motion. Equation (14) shows that the scattering function directly mirrors the longitudinal susceptibility $\chi_{11}^{\prime \prime} \sim 1 / C_{11}$ that relaxes with the stress relaxation. Thus low- $Q$ coherent scattering should observe the stress relaxation as it is exemplified by PCS results [35].

An independent derivation of Eq. (14) from linear response theory was given by Wang and Fischer [36]. Their work shows that Eq. (14) is more general, including also the second term from the entropy fluctuations, which was not considered here.

\section{EXPERIMENT}

\section{A. Samples}

The collective dynamics as well as the structure factor were studied on fully deuterated polyisobutylene ( $d$-PIB), while the self-motion was investigated on a protonated material ( $h$-PIB). The $h$-PIB was purchased from the American Polymer Standard Corp. (Mentor, OH). It has been synthesized by cationic polymerization.

The synthesis of $d$-PIB including the sources and the purification of materials has been described elsewhere [37]. Living cationic polymerization was carried out in a 500-ml three-neck flask equipped with an overhead stirrer at $-80^{\circ} \mathrm{C}$ under a dry $\left(\left[\mathrm{H}_{2} \mathrm{O}\right]<1.0 \mathrm{ppm}\right)$ nitrogen atmosphere in an MBraun 150-M glovebox. The target number average molecular weight $\left(M_{n}\right)$ deuteropolyisobutylene was 70000 . A 8.7-ml portion of a hexane (hex) stock solution $(0.2406 \mathrm{~g} / 25$ $\mathrm{ml}$ ) of the initiator, 5-tert-butyl-1,3-bis-(1-chloro-1methylethyl)benzene ( $t \mathrm{BuDiCumCl}), 0.263 \mathrm{ml}$ of 2,6-ditert-butylpyridine (DTBP), a proton trap, and $171 \mathrm{ml}$ of hex were charged to the flask at room temperature. The temperature was lowered to $-80^{\circ} \mathrm{C}$ and $94 \mathrm{ml}$ of methyl chloride $(\mathrm{MeCl})$ was added to reach the final ratio of hex/MeCl $=60 / 40(\mathrm{v} / \mathrm{v})$ at $-80^{\circ} \mathrm{C}$. Then $12.9 \mathrm{ml}$ of $\mathrm{TiCl}_{4}$ stock solution in $\mathrm{MeCl}\left(7 \mathrm{ml}\right.$ of $\mathrm{TiCl}_{4}$ in $\left.50 \mathrm{ml}\right)$ was added. Deuteroisobutylene was introduced to the flask in three equal increments of $8.8 \mathrm{ml}$ each, in $15 \mathrm{~min}$ intervals. The polymerization was quenched with prechilled methanol. After evaporation of the volatiles, $20.4 \mathrm{~g}$ of deuteropolyisobutylene (monomer conversion $=100 \%$ ) was obtained. Purification procedure of the product has already been reported [37].

Characterization. Concerning $h$-PIB, a molecular weight of $M_{w}=24900$ was inferred from gel permeation chromatography (GPC) agreeing well with light scattering data, that revealed $M_{w}=24200$. The number average molecular weight was obtained from vapor pressure osmometry yielding $M_{N}=19600$. The resulting value for $M_{w} / M_{N}=1.23$ agrees well with what can be deduced from GPC $\left(M_{w} / M_{N}\right.$ $=1.21$ ). The GPC trace shows a tail toward lower molecular weights with the $1 \%$ level reached at a molecular weight of about 7000 . The molecular weight of deuteropolyisobutylene
$\left(M_{n}=72000\right)$ and polydispersity $\left(M_{w} / M_{n}=1.05\right)$ were measured using a Waters high-performance liquid chromatography (HPLC) system equipped with a Model 510 HPLC pump, Model 410 differential refractometer, Model 486 tunable UV/Vis detector, on-line, multiangle laser light scattering (MALLS) detector (miniDawn, Wyatt Technology Inc.), Model 712 sample processor, and five Ultrastyragel GPC columns connected in the following series: $500,10^{3}, 10^{4}$, $10^{5}$, and $100 \AA$. The flow rate of tetrahydrofuran eluent was $1.0 \mathrm{ml} / \mathrm{min}$.

The perpendicular transmission $T_{\perp}$ measured with an incoming wavelength of $\lambda=6 \AA$ was $T_{\perp}=0.8$ for the $0.4-\mathrm{mm}$ $h$-PIB sample and $T_{\perp}=0.85$ for the 2 -mm $d$-PIB sample. Flat aluminum containers were used in all the experiments.

\section{B. Structure factor $S(Q)$}

The goal of this work was to study the intermediate-scale collective dynamics of PIB and to relate it to its self-motion. A prerequisite for this task is knowledge on the static structure factor $S(Q)$, in particular in the low and intermediate $Q$ regime, i.e., for $Q$ values around and below that corresponding to its first maximum. $S(Q)$ is defined as

$$
S(Q)=\frac{1}{N}\left\langle\sum_{i=1}^{N} \sum_{j=1}^{N} e^{i \mathbf{Q}\left(\mathbf{r}_{i}-\mathbf{r}_{j}\right)}\right\rangle,
$$

where $N$ is the total number of atoms in the sample, $\mathbf{r}_{i}$ and $\mathbf{r}_{j}$ the positions at $t=0$ of atoms $i$ and $j$, respectively. The brackets denote the ensemble average. In the following we will show that neutron scattering offers direct information on $S(Q)$ by measuring the differential cross section of fully deuterated samples. In the general case, the differential cross section contains two contributions, the coherent $\left(I_{\text {coh }}\right)$ and the incoherent one $\left(I_{\text {inc }}\right)$. This last contribution is $Q$ independent and its intensity is determined by the so-called incoherent cross section $\sigma_{\mathrm{inc}}$. The coherent contribution is related to the relative positions of the atoms in the sample,

$$
I_{\text {coh }}=\frac{1}{N}\left\langle\sum_{i=1}^{N} \sum_{j=1}^{N} b_{i} b_{j} e^{i \mathbf{Q}\left(\mathbf{r}_{i}-\mathbf{r}_{j}\right)}\right\rangle,
$$

where $b_{i}$ and $b_{j}$ are coherent scattering lengths of nuclei $i$ and $j$ respectively. Due to the nearly identical values of the coherent scattering lengths of carbon and deuterium, in the case of fully deuterated samples the coherent contribution to the differential cross section is just directly proportional to $S(Q)$, and its intensity is weighted by the coherent cross section $\sigma_{\text {coh }}=4 \pi \overline{b^{2}}$.

In this work we have performed three different experiments related to $S(Q)$ : (i) the intermediate and larger $Q$ regime was investigated using a neutron triple axis spectrometer (ii) the ratio between coherent and incoherent scattering was obtained by polarization analysis measurements at NSE spectrometers and (iii) the low $Q$ part of $S(Q)$ was studied by small-angle neutron scattering (SANS).

The triple axis spectrometer used was SV4 at the FRJ-2 research reactor in Jülich, Germany. Thereby, the instrument was used in a two axis mode, i.e., no energy analysis of the 
detected neutrons at a given scattering angle was made and consequently the differential cross section was measured. Pyrolithic graphite was used as monochromator (incoming neutron wave vector $k_{i}=2 \pi / \lambda=2.54 \AA^{-1}$ ). The data were corrected for background and geometrical factors.

Neutron polarization analysis allows us to determine the distinction between coherent and incoherent scattering. Spin incoherent scattering on protons leads for $\frac{2}{3}$ of the neutrons to a spin flip. Thus, for an upwardly polarized beam the scattering intensity becomes

$$
I_{\mathrm{up}}=I_{\mathrm{coh}}+\frac{1}{3} I_{\mathrm{inc}} .
$$

The intensity for the neutrons that have flipped their spin direction becomes

$$
I_{\text {down }}=\frac{2}{3} I_{\text {inc }},
$$

where $I_{\text {coh }}$ and $I_{\text {inc }}$ denote again the coherent and incoherent intensities. Using the spin echo spectrometers IN11C and IN15 at the Institut Laue-Langevin (ILL) in Grenoble, France, at incident wavelengths of $\lambda=6$ and $15 \AA$, respectively, such a polarization analysis has been performed at $T$ $=335,365$, and $390 \mathrm{~K}$.

The crucial low- $Q$ regime was investigated with the SANS instrument D22 at the ILL in Grenoble. The main issues are to obtain absolute intensities and to assess the amount of multiple scattering in the low- $Q$ regime. We studied the SANS from PIB for incoming neutron wavelengths between $\lambda=6$ and $18 \AA$ corresponding to $k_{i}$ values between $k_{i}=1.05$ and $0.34 \AA^{-1}$. The SANS detector was placed at $150 \mathrm{~cm}$ and a 2-m collimation section was used. The data were angularly averaged and corrected for empty cell scattering and background. Absolute calibration was performed with a 1-mm water cell that at $\lambda=7 \AA$ has an absolute cross section of $d \Sigma / d \Omega=0.918 \mathrm{~cm}^{-1}$. The wavelength dependence of the water scattering was accounted for by the empirical $g$ factor of Jacrot [38],

$$
g=\frac{1}{1-e^{-0.6 \sqrt{\lambda}}}
$$

The directional correction of the water transmission was calculated considering the angular dependence of the transmission

$$
T(\theta)=\frac{\exp \left[\frac{1}{\cos (\theta) \ln \left(T_{w}\right)}\right]}{T_{w}},
$$

where $\theta$ is the scattering angle and $T_{w}$ the straight water transmission.

\section{Dynamic experiments}

Neutron spin echo (NSE) is the most suitable technique for exploring dynamical processes in a wide momentum transfer $(Q)$ range with very high energy resolution. This is achieved by coding the energy transfer in the scattering process for each neutron individually into its spin rotation [39].
In this way, the application of precession magnetic fields before and after the scattering event results in a polarization of the neutron that depends only on the velocity difference of each neutron individually, irrespective of its initial velocity. Energy resolution and monochromatization of the incident beam are decoupled, and resolutions in energy of the order of $10^{-5}$ can be achieved with an incident neutron spectrum of $20 \%$ bandwidth.

The result of the NSE measurements is a normalized scattering function $\widetilde{S}_{\mathrm{NSE}}(Q, t)$ that contains the contributions from both the coherent and incoherent intermediate scattering functions,

$$
\widetilde{S}_{\mathrm{NSE}}(Q, t)=\frac{I_{\mathrm{coh}} \widetilde{S}_{\mathrm{coh}}(Q, t)-\frac{1}{3} I_{\mathrm{inc}} \widetilde{S}_{\mathrm{inc}}(Q, t)}{I_{\mathrm{coh}}-\frac{1}{3} I_{\mathrm{inc}}},
$$

where $\widetilde{S}_{\text {coh }}(Q, t)$ and $\widetilde{S}_{\text {inc }}(Q, t)$ are the normalized coherent and incoherent scattering functions (we are denoting with " " the functions that are normalized to their value at $t$ $=0$ ). Following the same reasoning as above for $S(Q)$, it is followed that in a fully deuterated sample the coherent scattering function is equivalent to the true pair (collective) correlation function: $\widetilde{S}_{\text {coh }}(Q, t) \equiv S_{\text {coh }}(Q, t) / S(Q)=\widetilde{S}_{\text {pair }}(Q, t)$. In our case, the coherent and incoherent intensities of $d$-PIB are known experimentally from the polarization analysis results up to a common factor (see Sec. IV A). These experimental results agree well with the expectation from the known cross sections and the results on the structure factor $S(Q)$. On the other hand, we note that in the low $Q$ limit the pair correlation function averages over large regions of the sample and is determined by the density-density correlation function

$$
\frac{S_{\text {pair }}(Q, t)}{S(Q)}=\frac{\left\langle\Delta \rho^{*}(Q, t) \Delta \rho(Q, 0)\right\rangle}{\left\langle\Delta \rho^{*}(Q) \Delta \rho(Q)\right\rangle},
$$

where $\Delta \rho(Q, t)$ is the Fourier component of the density fluctuations corresponding to the wave vector $Q$ and the time $t$. The $*$ denotes the complex conjugate and the pointed brackets signify the thermal average.

In the case of the protonated sample, due to the large value of the incoherent cross section of the proton $\sigma_{\text {inc }}^{H}$ compared to all the other cross sections, $I_{\text {inc }} \gg I_{\text {coh }}$ whatever the modulation of $I_{\text {coh }}$ due to the $Q$-dependent partial structure factor will be. Moreover, $\sigma_{\text {inc }}^{C}=0$ and thus the incoherent signal corresponds to the self-correlation function of the protons $\widetilde{S}_{\text {inc }}(Q, t)=\widetilde{S}_{\text {self }}(Q, t)$. From the NSE signal it is then possible to obtain information on the self-motion of the $\mathrm{H}$ atoms when using protonated samples. However, we note the difficulties to observe the self-motion by NSE. The spin flip scattering that was discussed in relation with Eqs. (17) and (18) reduces the spin echo amplitude immediately by a factor of 3 and, in addition, creates a large background. Furthermore, since incoherent scattering leads to a negative echo amplitude, any small amount of coherent scattering adds with a positive sign and easily diminishes the amplitude to zero. On the other hand, multiple scattering contributions are 
less severe, since each additional scattering process reduces the amplitude by another factor of 3 .

In an NSE experiment the time variable depends on the precession field $B$, the wavelength $\lambda$ and the length of the precession field $L: t \sim B L \lambda^{3}$. Thus, the time evolution of the correlation function accessed can be followed in varying $B$ and $\lambda$.

This work was carried out at the multidetector NSE instrument IN11C at the ILL in Grenoble using two different initial wavelengths $\lambda=6$ and $10 \AA$. Supporting experiments were performed at the high-resolution long-wavelength instrument IN15 also at the ILL.

The multidetector at IN11C covers an angular range of $30^{\circ}$ in the horizontal plane. For the $6-\AA$ measurements it was placed at $32^{\circ}, 65^{\circ}$, and $85^{\circ}$ scattering angle for its central detector. Thereby, a $Q$ range $0.31 \leqslant Q \leqslant 1.6 \AA^{-1}$ and a time range $8 \mathrm{ps} \leqslant t \leqslant 1.7 \mathrm{~ns}$ were explored. The measurement at 10 $\AA$ was used in order to extend the $Q$ and time regime towards lower $Q$ and longer times. Here, the detector was placed at $32^{\circ}$ accessing a minimum $Q=0.20 \AA^{-1}$ and a maximum Fourier time $t_{\max }=5.5 \mathrm{~ns}$. All these experiments were performed at the temperatures $T=335,365$, and $390 \mathrm{~K}$ with exception of the $10-\AA$ measurements on the self-motion, where only $T=390 \mathrm{~K}$ was investigated. In the case of the collective dynamics the high-resolution instrument IN15 was used at a wavelength $\lambda=15 \AA$ above the multiple scattering (MS) threshold, in order to control the influence of MS contributions. These experiments were done at $T=310,335$, and $365 \mathrm{~K}$.

For each momentum transfer the instrumental resolution function was determined from the elastic scattering of the sample at $4 \mathrm{~K}$ (larger $Q$ ) or from a TiZr alloy (lower $Q$ ). The instrumental background from the cryofour and the aluminum container were measured separately and subtracted from the experimental spectra using the appropriate transmission factors. The background-corrected spectra were divided by the resolution function revealing the normalized intermediate dynamic structure factors.

\section{RESULTS}

\section{A. Structure factor}

Figure 1 displays the static structure factor at $T=320 \mathrm{~K}$ as obtained by SV4. $S(Q)$ is characterized by a first strong peak at around $Q=1 \AA^{-1}$ and a broad second peak in the $Q$ regime $1.7 \leqslant Q \leqslant 3.4 \AA^{-1}$. The scatter of data points between 2.5 and $3 \AA^{-1}$ relates to the Al-Debye-Scherrer rings of the sample container, which could not be fully subtracted. Above the glass-transition temperature $T_{g}=205 \mathrm{~K}$, the first peak is observed to shift with temperature with a linear expansion coefficient $\beta=5.0 \times 10^{-4} \mathrm{~K}^{-1}$. This large expansion coefficient identifies the nature of the first peak as resulting from interchain correlations. Its position at $Q_{\max } \cong 1 \AA^{-1}$ marks a relatively large interchain distance of $d_{\text {chain }}=2 \pi / Q_{\max }$ $\cong 6.3 \AA$. The second peak is little affected by temperature and must relate to a large extent to interchain correlations governed by covalent bonds.

The observed $S(Q)$ profile sits on a relatively high background which relates partly to incoherent partly to multiple

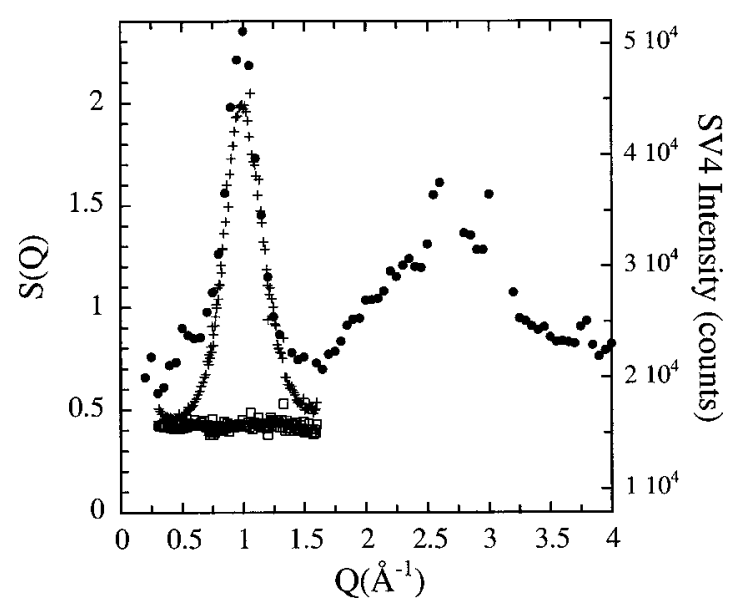

FIG. 1. Differential scattering cross section measured for PIB by means of the triple axis spectrometer SV4 (). The corresponding scale (right) is chosen so that the incoherent contribution coincides with the origin for the static structure factor scale (left). NSE spectrometer IN11C results on the static structure factor are shown for comparison $(+)$ as well as the incoherent contribution measured also by IN11C ( $\square$ ) (scale on the left).

scattering. The polarization analysis performed by IN11C allowed to determine the coherent and incoherent contributions of the scattering. The outcome of this analysis is also displayed in Fig. 1, where the open squares denote the incoherent and the crosses the coherent intensities at $335 \mathrm{~K}$. We now assume that the ratio of coherent and incoherent scattering for the triple axis and the NSE machine are identical. Furthermore, we consider the different resolution conditions at the triple axis $(\Delta \lambda / \lambda \cong 1 \%)$ and $\operatorname{NSE}(\Delta \lambda / \lambda \cong 20 \%)$ instruments-their main effect is a reduction of the peak height at the NSE by $17 \%$. With the known $I_{\text {coh }} /\left(I_{\text {coh }}\right.$ $\left.+I_{\text {inc }}\right)$ ratios we now are able to define the incoherent baseline for the triple axis measurement to $\approx 7900$ counts. With the intensity scaling in place the coherent and incoherent NSE data are placed in Fig. 1 "up to scale." Finally, the $S(Q)$ scale is adjusted such as to place the " 1 " as the line around which the oscillations of the second peak take place.

We now concentrate on the low- $Q$ regime. The sequence of a low intensity level at low $Q$ followed by an intense first halo or first structure factor peak makes multiple scattering a serious problem in the low- $Q$ regime of liquid and glasses. Intense secondary scattering may arise from the first halo and add significantly to the low primary scattering. For example, recently, for CKN, Russina et al. [40] have estimated a $400 \%$ contribution of MS in the low- $Q$ regime of this material. This type of MS may be avoided if the incoming neutron wave vector $k_{i}=2 \pi / \lambda$ is smaller than $Q_{\max } / 2$. Then the Ewald sphere does not touch the first halo and this source of MS is eliminated.

The coherent to incoherent ratio measured by both IN11 and IN15 spectrometers was found to be close to one in the low- $Q$ range. Thus, half of the intensity was coherent. Figure 2 displays the thus evaluated coherent intensities for the different incident neutron wavelengths used. The observed SANS intensities are highest for $\lambda=10 \AA$. This result may be rationalized from the largest amount of MS that results 


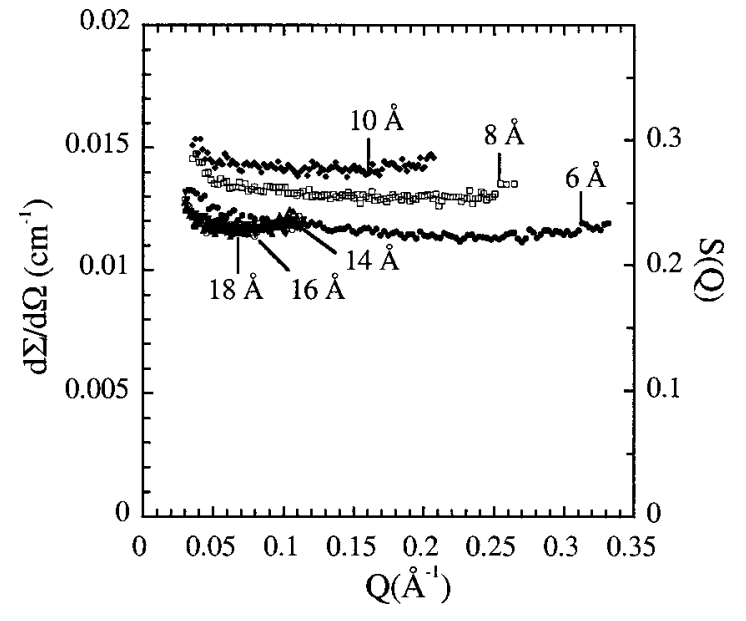

FIG. 2. Differential scattering cross section measured for PIB by means of the SANS instrument D22. The different symbols correspond to the different incoming wavelengths indicated. From the scale on the right the corresponding values of $S(Q)$ can be read.

from the amorphous halo at about $\theta=90^{\circ}$. Going away from this condition reduces the MS and thus the results at $\lambda=8$ and $6 \AA$ fall below the $\lambda=10 \AA$ results. For the wavelengths $\lambda \geqslant 14 \AA$ the first halo is not excited and MS is expected to be minimal. There the absolute cross section amounts to $d \Sigma / d \Omega=0.0115 \mathrm{~cm}^{-1}$. The data thus nicely demonstrate the role of the first halo in the MS at low $Q$ in a liquid. They also show that the MS contribution at wavelengths close to $10 \AA$ amounts to about $30 \%$. Thus, other than in the case of CKN, the signal at a low $Q$ is still dominated by single scattering events.

We may convert the observed absolute cross section to the structure factor. For this purpose we have to divide by the average cross section per average atom volume $N_{a} \overline{b^{2}} / \bar{v}$, where $N_{a}$ is the Avogadro number, $\overline{b^{2}}=\left(4 b_{C}^{2}+8 b_{D}^{2}\right) / 12$ with $b_{C}$ and $b_{D}$ the scattering length of $C$ and $D$, respectively, and $\bar{v}$ the average molar volume per atom. This procedure leads to

$$
S(Q \rightarrow 0)=(0.22 \pm 10) \%
$$

at room temperature. The error is an estimate for possible systematic errors rather than the statistical error.

\section{B. Self-motion}

Figure 3 displays a set of NSE spectra taken from $h$-PIB at $T=390 \mathrm{~K}$ for a number of different $Q$ values. In going from larger to smaller $Q$ the relaxation process is continuously slowed down. For the smallest $Q \approx 0.48 \AA^{-1}$ also data taken at $\lambda=10 \AA$ are shown.

The data were evaluated following a two step procedure. In the first step all spectra were fitted with a stretched exponential or Kohlrausch-Williams-Watts (KWW) function, given by the general expression

$$
\widetilde{S}(Q, t)=A(Q) \exp \left\{-\left[\frac{t}{\tau(Q)}\right]^{\beta}\right\},
$$

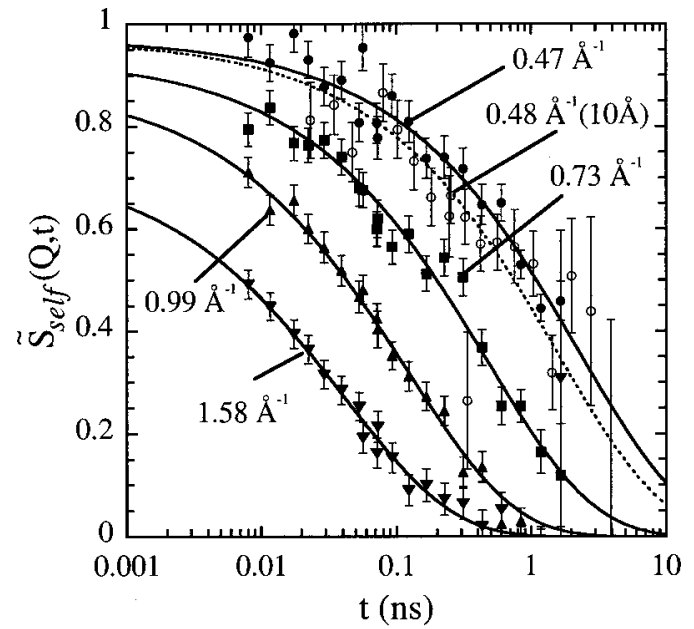

FIG. 3. Time evolution of the self-correlation function measured on a fully protonated PIB sample by means of IN11C. The symbols (full: incoming wavelength $\lambda=6 \AA$; empty: $\lambda=10 \AA$ ) correspond to the different $Q$ values indicated. Lines are the resulting KWW fit curves [Eq. (24)] (solid: $\lambda=6 \AA$; dotted: $\lambda=10 \AA$ ).

where $A(Q)$ is the amplitude, $\beta$ is the stretching exponent and $\tau(Q)$ is a generic relaxation time. In this work we deal with a number of different relaxation times depending on the particular correlation function investigated. For clarity we would like to define them now. With $\tau_{\text {self }}$ and $\tau_{\text {pair }}$ we denote the characteristic times obtained from the self- and paircorrelation functions, $\widetilde{S}_{\text {self }}(Q, t)$ and $\widetilde{S}_{\text {pair }}(Q, t)$, respectively. In order to emphasize the particular importance of the structural relaxation time that is measured at the structure factor maximum we define $\tau_{\mathrm{st}} \cong \tau_{\text {pair }}\left(Q_{\max }\right)$. For the discussion a comparison with the relaxation time of the mechanical stress will be of great importance. This stress relaxation time is $\tau_{M}$.

In the first fitting of $\widetilde{S}_{\text {self }}(Q, t)$ by means of Eq. (24), the amplitude and the characteristic time were the fitting parameters, whereas the value of $\beta$ was fixed at 0.55 , that obtained in earlier measurements for the relaxation at $S\left(Q_{\max }\right)$ [25]. While at the structure factor maximum the older study firmly established $\beta$, the present measurements do not allow an independent fit of $A_{\text {self }}(Q), \tau_{\text {self }}(Q)$, and $\beta$.

For the incoherent spectra the amplitude parameters decrease continuously with increasing momentum transfer following a Debye-Waller factor

$$
A_{\text {self }}(Q)=\exp \left(-\left\langle u^{2}\right\rangle Q^{2} / 3\right)
$$

where $\left\langle u^{2}\right\rangle$ is the average mean square displacement of the proton in motions faster than the relaxation process considered here. A fit with Eq. (25) to the observed $A_{\text {self }}(Q)$ yielded $\left\langle u^{2}\right\rangle=0.375 \AA^{2}$ that did not vary with temperature within the experimental accuracy. In the next step $A_{\text {self }}(Q)$ was fixed to the respective values prescribed by the Debye-Waller factor and $\tau_{\text {self }}(Q)$ was refined.

The upper part of Fig. 4 displays the $Q$ - and temperaturedependent results. As guide to the eye solid lines mark the $Q^{-2 / \beta}$ power law 


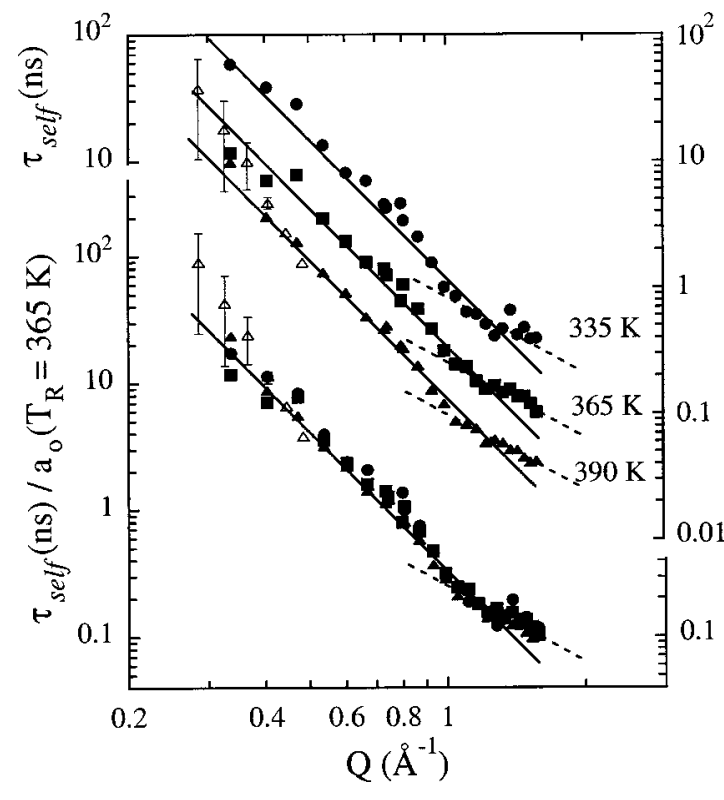

FIG. 4. Upper part: Momentum transfer dependence of the characteristic time of the KWW functions describing the self-correlation function at 335 (circles), 365 (squares), and $390 \mathrm{~K}$ (triangles). Lower part: Scaling representation: 335 and $390 \mathrm{~K}$ data have been shifted to the reference temperature $365 \mathrm{~K}$ applying a shift factor corresponding to an activation energy of $0.43 \mathrm{eV}$. Full symbols correspond to results from measurements performed with incoming wavelength $\lambda=6 \AA$ and empty symbols to $\lambda=10 \AA$. Solid (dotted) lines through the points represent $Q^{-2 / 0.55}\left(Q^{-2}\right)$ power laws.

$$
\tau_{\text {self }}(Q)=a_{\text {self }}(T) Q^{-2 / \beta}
$$

expected for dynamics in Gaussian approximation [41,42] (see also later). The prefactor $a_{\text {self }}(T)$ reflects the temperature dependence of the characteristic time in the power law relation. In the $Q$ regime $Q \leqslant 1 \AA^{-1}$ the $Q$ dependence of all data sets is well represented by such an asymptote. At $Q$ values slightly above $Q=1 \AA^{-1}$ a tendency towards a crossover leading to a weaker power law for $\tau_{\text {self }}(Q)$ is apparent. The dotted lines indicate a $Q^{-2}$ law. Indications of a similar crossover in the $Q$ dependence have been recently reported by Colmenero, Alvarez, and Arbe [43] from molecular dynamics simulations on polyisoprene. However, in our case for a firm determination of a power law above $1 \AA^{-1}$ the $Q$ range is much too small.

Looking on the temperature dependence we realize that the rheological shift factor $a_{F}$ established by Ferry [20],

$$
\ln \left[a_{F}(T)\right]=-\frac{20.91(T-298.2)}{209+T-298.2},
$$

does not superimpose the data [44]. This is in contrast to a recent NSE-investigation that had demonstrated that this shift factor accounts very well for the temperature dependence of the collective response at the first structure factor maximum [25]. Unexpectedly, they follow a weaker temperature dependence with an activation energy $E_{o}$ $=0.43 \mathrm{eV}$, intermediate between the activation energy of the $\beta$ process $\left(E_{\beta}=0.26 \mathrm{eV}\right)$ and the apparent activation energy

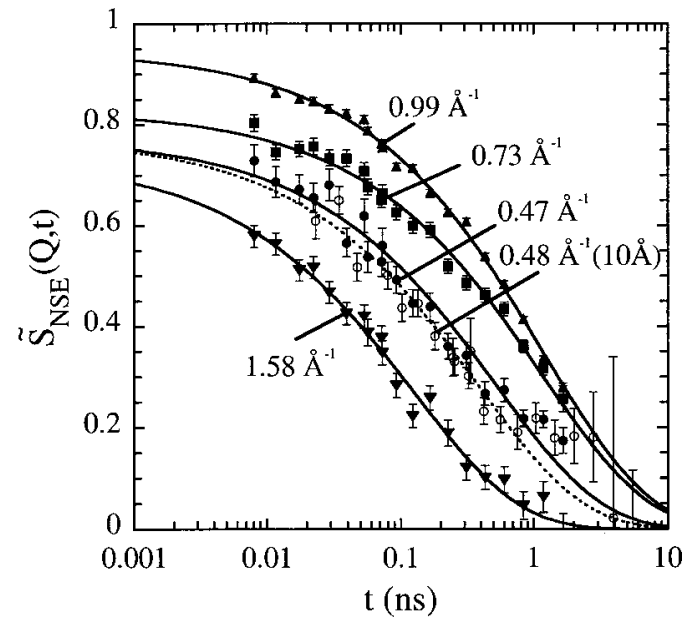

FIG. 5. Dynamic structure factor measured by IN11C on the deuterated PIB sample. The symbols (full: incoming wavelength $\lambda=6 \AA$; empty: $\lambda=10 \AA$ ) correspond to the different $Q$ values indicated. Lines are the resulting KWW fit curves [Eq. (24)] (solid: $\lambda=6 \AA$; dotted: $\lambda=10 \AA$ ).

of the viscosity in our temperature regime $E_{\alpha}=0.67 \mathrm{eV}$. The lower part of Fig. 4 displays the such shifted data and we realize that the weaker temperature dependence holds over the full $Q$ range.

\section{Collective motion}

The data on the collective dynamics were obtained from the $d$-PIB sample using the setups and procedures described in Sec. II C. Figure 5 displays selected spectra $\widetilde{S}_{\mathrm{NSE}}(Q, t)$ at $390 \mathrm{~K}$ from different regions of the structure factor:

(i) from the low- $Q$ plateau, data at $Q$ around $0.47 \AA^{-1}$ are shown that were taken with incident wavelengths of 6 and 10 $\AA$. In spite of the low scattered intensity still a reasonable statistics is achieved. Furthermore, it is satisfying to observe the good agreement between the results from the two different experimental setups.

(ii) The weakest relaxation is observed for the spectrum at $Q \approx 1.0 \AA^{-1}$ at the structure factor peak, while (iii) the fastest relaxation is detected at $Q=1.58 \AA^{-1}$, close to the first minimum of $S(Q)$. (iv) The spectrum at $Q=0.73 \AA^{-1}$ in the lower $Q$ flank of $S(Q)$ relaxes at a rate intermediate between that of the low- $Q$ plateau and that of the structure factor peak. Finally, we note that in all cases a nearly full relaxation of $\widetilde{S}_{\mathrm{NSE}}(Q, t)$ within the instrumental time frame is observed.

In order to assess possible effects of multiple scattering we compare NSE spectra taken above and below the MS threshold established by small-angle neutron scattering. Figure 6 displays IN15 and IN11C data taken at $\lambda=10$ and 15 $\AA$, respectively. Though the data have quite some statistical scatter-carrying out experiments at these long wavelengths at a cross section of $\approx 0.01 \mathrm{~cm}^{-1}$ is very difficult-in both cases within the statistical error the respective data sets taken above and below the MS threshold agree very well. Thus, we prove experimentally that for the intermediate length scale dynamics at low $Q$ the MS effects on the dynamics may be ignored within our level of accuracy. 


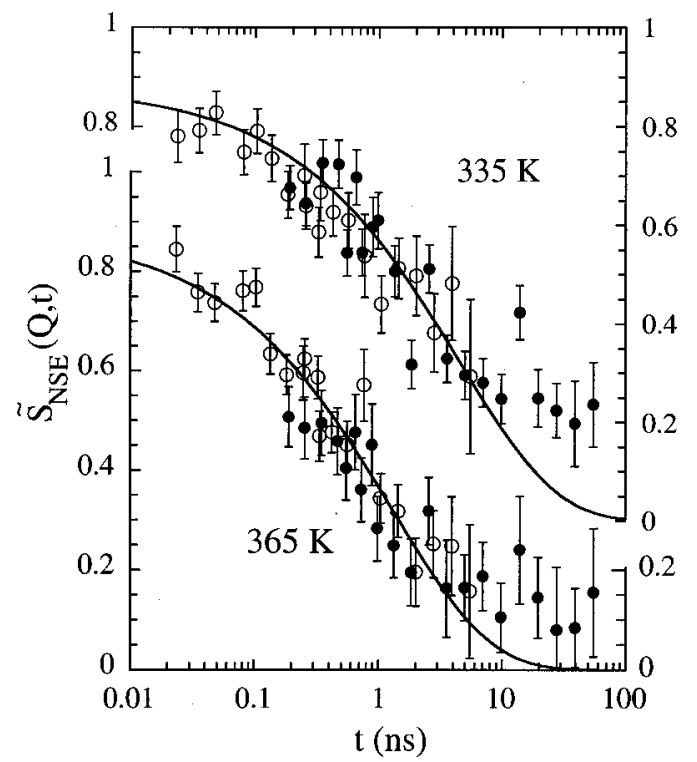

FIG. 6. Dynamic structure factor of PIB measured for $Q$ $=0.2 \AA^{-1}$ by IN11C with an incident wavelength of $\lambda=10 \AA$ (open symbols) and IN15 with $\lambda=15 \AA$ (full symbols) at the two temperatures indicated.

An evaluation of the data from $d$-PIB must consider the incoherent scattering from deuterium atoms $(2 \mathrm{~b}$ incoherent compared to $5 \mathrm{~b}$ coherent). Adding the atomic scattering cross sections of one monomer we arrive at $60 \mathrm{~b}$ coherent and $16 \mathrm{~b}$ incoherent total cross sections. In the low- $Q$ part, where the coherent structure factor is low, the incoherent scattering will contribute importantly (see Fig. 1). Solving Eq. (21) for $\widetilde{S}_{\text {coh }}(Q, t) \equiv \widetilde{S}_{\text {pair }}(Q, t)$ we get

$$
\begin{aligned}
\widetilde{S}_{\text {pair }}(Q, t)= & \frac{S_{\text {pair }}(Q, t)}{S(Q)}=\frac{I_{\text {coh }}-\frac{1}{3} I_{\text {inc }}}{I_{\text {coh }}} \widetilde{S}_{\text {NSE }}(Q, t) \\
& +\frac{1}{3} \frac{I_{\text {inc }}}{I_{\text {coh }}} \widetilde{S}_{\text {self }}(Q, t) .
\end{aligned}
$$

Equation (28) defines the correction procedure leading to the desired pair correlation function. For $\widetilde{S}_{\text {self }}(Q, t)$ we introduce interpolated functions with characteristic relaxation times from the solid lines in the upper part of Fig. 4. The correction is only important in the low- $Q$ regime, where $S(Q)$ is small. There the contribution of $\widetilde{S}_{\text {self }}(Q, t)$ amounts to about $23-$ $28 \%$ depending on temperature. Figure 7 displays, for spectra taken at $\lambda=6$ and $10 \AA$, the magnitude of the correction. By the addition of a fraction of the incoherent scattering function the data points rise and the corresponding collective relaxation time increases.

The such corrected data were fitted to Eq. (24) varying both the amplitude $A_{\text {pair }}(Q)$ and the relaxation time $\tau_{\text {pair }}(Q)$. The stretching exponent $\beta$ was fixed again at 0.55 .

Figure 8 displays the $Q$-dependent amplitudes obtained by this fitting process. We observe a well-defined maximum of the amplitude at $Q_{\max }$ with a $Q$-dependence mirroring the structure factor. This is demonstrated by a superposition with the coherent intensity $\sim S(Q)$ obtained from the polarization

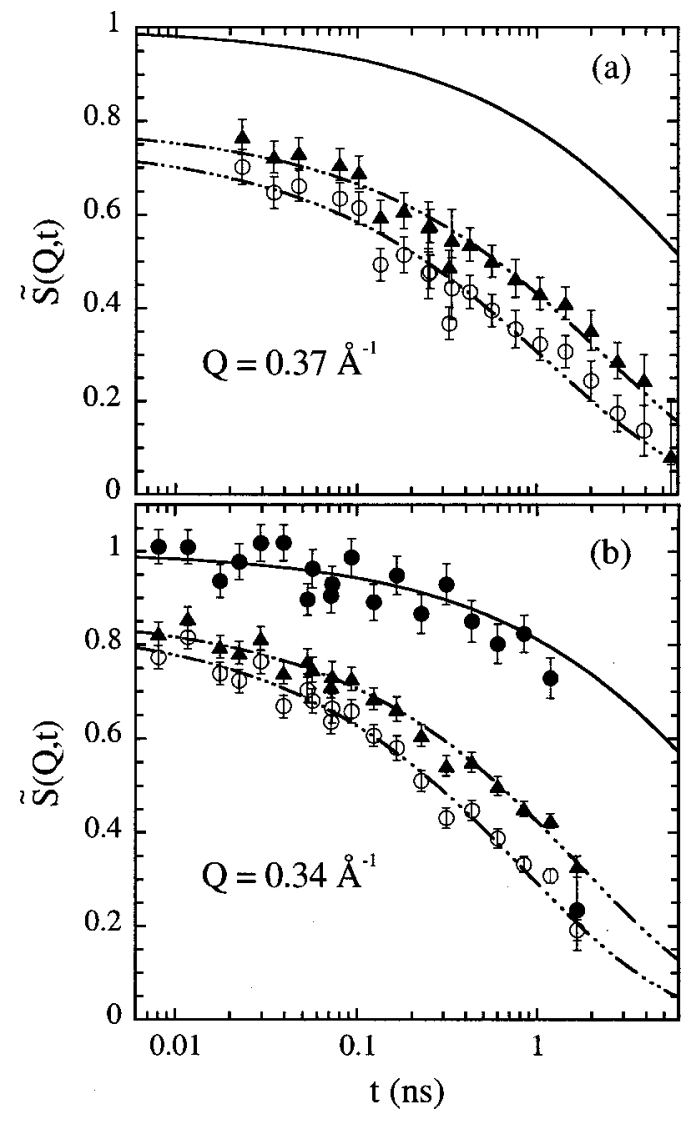

FIG. 7. Comparison between the incoherent scattering function $\widetilde{S}_{\text {self }}(Q, t)$ ( experimental data; solid line: interpolated function), the experimental functions measured on the deuterated PIB sample $\widetilde{S}_{\mathrm{NSE}}(Q, t)(\bigcirc)$, and the purely coherent scattering function $\widetilde{S}_{\text {pair }}(Q, t)(\mathbf{\Delta})$ calculated by means of Eq. (28) for two $Q$ values close to $0.35 \AA^{-1}$ at $365 \mathrm{~K} ; \lambda=10 \AA$ (a) and $\lambda=6 \AA$ (b). Dasheddotted lines show the fitting curves corresponding to KWW functions with $\beta=0.55$.

analysis at IN11C. Reflecting the lower statistics of the data at low $Q$ there the amplitude scatters around an average value. This value decreases with increasing temperature, from $\approx 0.86$ at $335 \mathrm{~K}$ to $\approx 0.8$ at $390 \mathrm{~K}$. $\left(1-A_{\text {pair }}\right)$ is the contribution of the fast process $[1,2]$ in the dynamics of glass-forming liquids to the dynamic structure factor. The strong minimum of $\left(1-A_{\text {pair }}\right)$ at $Q_{\max }$ relates to the de Gennes narrowing [7] of the fast process and is also well known in the framework of the MCT $[1,2]$.

Figure 9(a) presents the thus obtained $Q$-dependent relaxation times for the different temperatures and incident wavelengths. For all three temperatures we observe the same general behavior with the collective relaxation times displaying a low- $Q$-plateaulike behavior with some tendency of an increase towards the lowest $Q$. The low- $Q$ behavior is followed by an increase of the relaxation times to a maximum value that shifts from about $Q=0.8 \AA^{-1}$ at $T=390 \mathrm{~K}$ to $Q$ $=1 \AA^{-1}$ at $T=335 \mathrm{~K}$. Towards higher $Q$ a strong decrease of the relaxation times is observed. The peak height relative to the low- $Q$ plateau is increasing with decreasing temperature. While at $T=390 \mathrm{~K}$ the relative peak height amounts to about a factor of 2, this changes to 4 at $335 \mathrm{~K}$. 


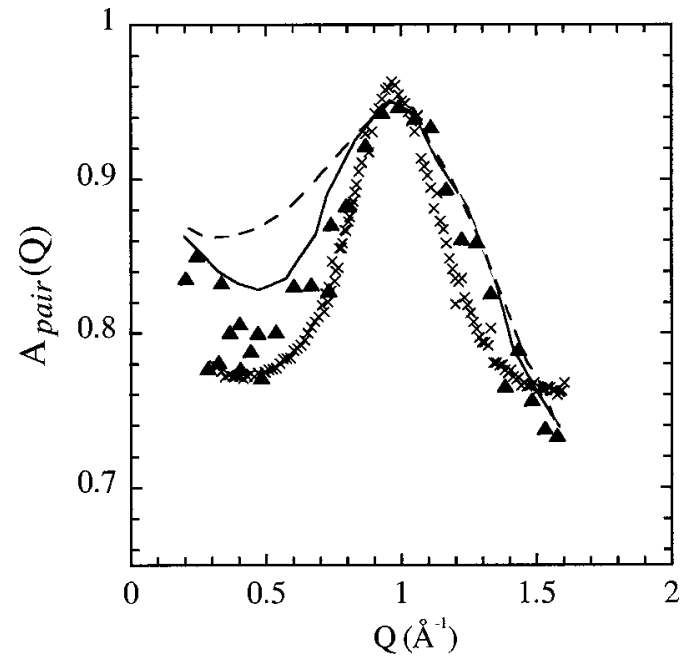

FIG. 8. Momentum transfer dependence of the amplitude of the KWW functions describing the dynamic structure factor. For $390 \mathrm{~K}$ all the values obtained are shown $(\mathbf{\Delta})$, while the dashed and solid line represent the smoothed behavior for 335 and $365 \mathrm{~K}$, respectively. The static structure factor as obtained with IN11C is shown in arbitrary units for comparison $(\times)$.

Comparing the time scales with those from the selfmotion, e.g., at the $Q$ value of the static structure maximum $Q_{\max }=1 \AA^{-1}$ we find a more-than-one order of magnitude faster relaxation of the self-correlation function compared to that of the collective response $\left[\tau_{\text {self }}\left(Q_{\max }\right)=0.07 \mathrm{~ns}\right.$; $\tau_{\text {pair }}\left(Q_{\max }\right)=\tau_{\mathrm{st}}=1 \mathrm{~ns}$ at $\left.390 \mathrm{~K}\right]$. From this qualitative observation we may immediately conclude that the relaxation times in the peak region close to the structure factor maximum shift differently with temperature than in the low- $Q$ regime-a rather unexpected result. Figures $9(\mathrm{~b})-(\mathrm{c})$ investigate the temperature dependence in more detail. In Fig. 9(b) the observed relaxation times are shifted with the rheological shift factor [Eq. (27)] due to Ferry. This figure corroborates our earlier result that at $Q \geqslant Q_{\max }$ the rheological shift factor describes well the temperature dependence of the collective response. For $Q \geqslant 1 \AA^{-1}$ the shift factor of Eq. (27) establishes a master curve indicating that here the temperature dependence agrees well with the viscosity results. On the low- $Q$ side, on the other hand, severe discrepancies evolve. Obviously, the data do not follow the temperature dependence of the viscosity.

Figure 9(c) investigates the temperature dependence of the $\beta$ process that in PIB is characterized by an activation energy of $E_{\beta}=0.26 \mathrm{eV}$ [25]. Such a shifting procedure leads to grossly wrong results at higher $Q$, while in the lower- $Q$ regime the relaxation rates are coming closer together than before. While $a_{F}$ overestimates the temperature dependence of $\tau_{\text {pair }}(Q)$, the $\beta$ process is too weakly dependent on temperature.

In order to proceed further we analyzed the temperaturedependent results in terms of an Arrhenius law and fitted this law to the relaxation times at each $Q$-value separately, the fitting parameter being the activation energy. Figure 10 displays the so obtained $Q$-dependent activation energies. Horizontal lines indicate the effective activation energy of the

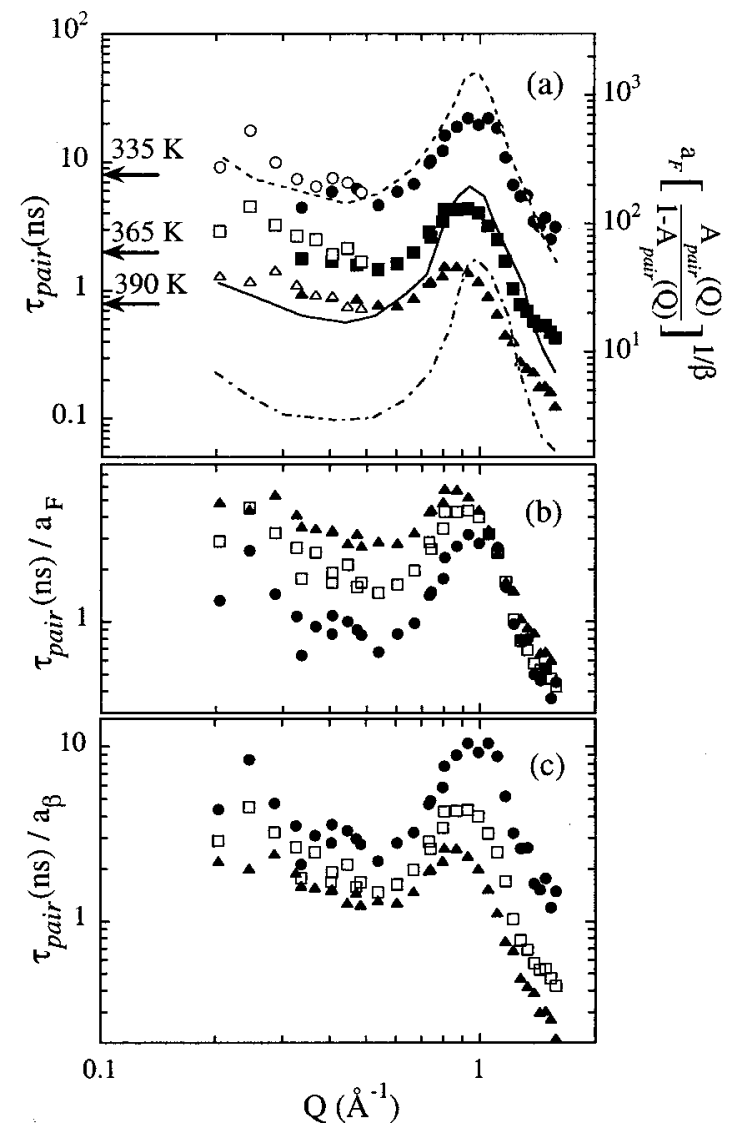

FIG. 9. Momentum transfer dependence of the characteristic time of the KWW functions describing the dynamic structure factor at 335 (circles), 365 (squares), and $390 \mathrm{~K}$ (triangles). Figure (a) shows the values obtained for each temperature. Taking $365 \mathrm{~K}$ as reference temperature, the application of the rheological shift factor to the times gives figure (b) and using the shift factor deduced from the temperature dependence of the dielectric $\beta$-relaxation figure (c) is obtained. In (a) full symbols correspond to results obtained with $\lambda=6 \AA$, empty symbols to $\lambda=10 \AA$. The arrows show the interpolated mechanical susceptibility relaxation times at the temperatures indicated. The lines display the $Q$ dependence of the characteristic times predicted by MCT [Eq. (35) affected by the rheological shift factor] for 335 (dashed), 365 (solid), and $390 \mathrm{~K}$ (dashed-dotted).

viscosity in the temperature range of observation $\left(E_{\alpha}\right.$ $=0.67 \mathrm{eV}$ ) and of the $\beta$ processes, $E_{\beta}=0.26 \mathrm{eV}$. The experimental $Q$-dependent activation energies reach $E_{\alpha}$ around $Q_{\max }$ and decrease to an average value of $E_{o} \approx 0.42 \mathrm{eV}$ in the low- $Q$ regime. We note that this intermediate value agrees perfectly with the activation energy found for the temperature shift of the self-motion (see the lower part of Fig. 4). At higher $Q$ beyond the first static structure factor peak the activation energies are slightly smaller than $E_{\alpha}$ indicating an admixture of the $\beta$ relaxation (see also Ref. [25]). We thus substantiate that the low- $Q$ collective relaxation does not resemble the viscosity but follows a significantly weaker temperature dependence.

We note, and we will come back to this later, that the observed activation energy of $0.42 \mathrm{eV}$ coincides with the activation energy of an intrachain relaxation process, which limits the validity of the Rouse model towards shorter length 


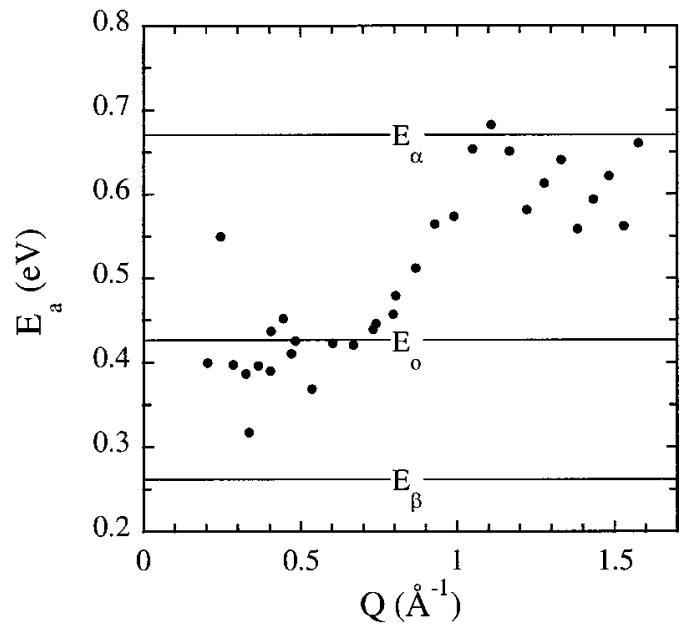

FIG. 10. Momentum transfer dependence of the activation energy observed for the collective dynamics. Horizontal lines show the values obtained from the rheological shift factor in the temperature range investigated $\left(E_{\alpha}\right)$, for the dielectric $\beta$ relaxation $\left(E_{\beta}\right)$, and for the time characteristic for the intrachain viscosity $\left(E_{o}\right)$.

scales in this polymer [6]. As will be pointed out in the discussion, the same apparent activation energy regulates the temperature shift of the sound waves damping in the relevant frequency range between 1 [24] and $5 \mathrm{GHz}$ [23]. At these high frequencies, the viscosity shift factors no longer agree with the stress relaxation shift factors; the latter show a lower apparent activation energy of $0.48 \mathrm{eV}$, close to the low- $Q$ relaxation value.

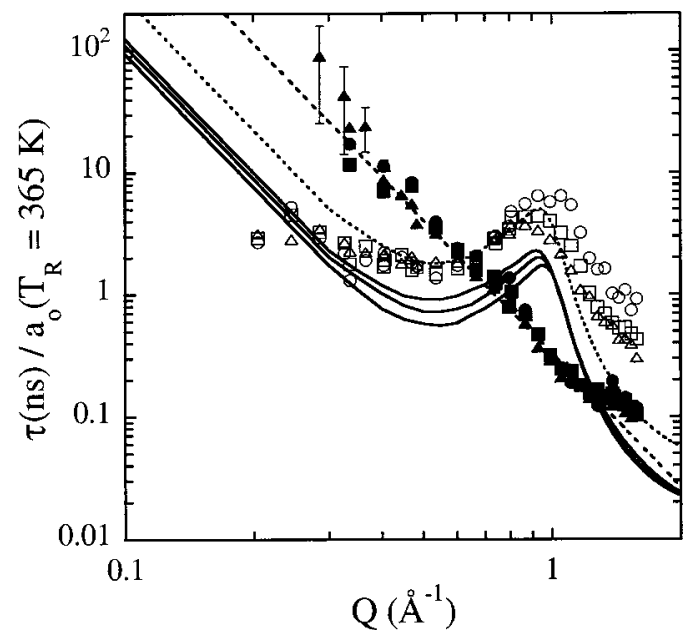

FIG. 11. Result of applying the shift factors corresponding to an activation energy of $0.43 \mathrm{eV}$ to the relaxation times observed for the collective dynamics (empty symbols) and the self-correlation (full symbols): 335 (circles), 365 (squares), and $390 \mathrm{~K}$ (triangles). The reference temperature is $365 \mathrm{~K}$. Dotted line through the selfcorrelation data shows the $Q^{-2 / 0.55}$ dependence implying Gaussian behavior. Solid lines show the results from the Sköld ansatz [Eq. (34)] when using the $S(Q)$ corresponding to 390,365 , and $335 \mathrm{~K}$ (from top to bottom). The dashed-dotted line is obtained by shifting the solid one for $365 \mathrm{~K}$ in order to match the corresponding coherent experimental points (multiplied by a factor of 2.5 ).
Figure 11 summarizes the obtained results on the temperature behavior and displays the collective relaxation times together with that characteristic for the proton self-motion, all shifted with the intermediate temperature law $\left(E_{o}\right.$ $=0.43 \mathrm{eV}$ ). It is evident that the temperature dependence of the collective motion for $Q \leqslant 0.7 \AA^{-1}$ and that of the selfmotion over the full $Q$ range are very well described by the intermediate temperature law. While the characteristic times for self-motion increase with a power law $\tau_{\text {self }} \approx Q^{-2 / \beta}$ towards low $Q$, the collective times display only a weak $Q$ dependence and thus $\tau_{\text {pair }}$ and $\tau_{\text {self }}$ separate from each other the smaller the $Q$ becomes.

\section{DISCUSSION}

\section{A. Structure factor}

The primary result of our study comes from the low- $Q$ SANS experiments. The wavelength-dependent measurements revealed absolute intensities and allowed to experimentally separate multiple from single scattering contributions. It was shown that MS amounts to only about $30 \%$ of the total scattering in this critical low- $Q$ regime.

The structure factor in the low- $Q$ regime, $S(Q \rightarrow 0)$ $\approx 0.22$, at room temperature compares well with the value of 0.183 obtained from Eq. (3). As outlined in Sec. II, $80 \%$ of that value comes from the adiabatic compressibility and only $20 \%$ from entropy fluctuations. The adiabatic compressibility term should decay with the stress relaxation time, independent of $Q$, while the entropy fluctuations are expected to decay with the thermal conductivity time

$$
\tau_{\text {th }}=\rho C_{p} Q^{-2} / \lambda_{\text {th }},
$$

where $\lambda_{\text {th }}$ is the thermal conductivity $\left(\lambda_{\text {th }}\right.$ $=0.13 \mathrm{~J} \mathrm{~s}^{-1} \mathrm{~m}^{-1} \mathrm{~K}^{-1}$ for PIB at room temperature). For polyisobutylene at room temperature, the values [32] yield an entropy relaxation time of $0.013 \mathrm{~ns}$ at $0.1 \AA^{-1}$; this time should depend only weakly on temperature and its $Q$ dependence should saturate at some $Q$-value that could be estimated to be of the order of $1 \mathrm{~nm}^{-1} \cdot \tau_{\mathrm{th}}$ is at the lower bound of our observation window, i.e., the corresponding decay occurs at times shorter than the observation range and therefore will manifest itself by a reduced amplitude factor $A_{\text {pair }}$. The observation of $A_{\text {pair }} \approx 0.8$ at low $Q$ agrees well with the estimated $20 \%$ contribution of the heat conduction process to $\widetilde{S}_{\text {pair }}(Q, t)$.

Considering systematic errors of about $10 \%$ most of the observed intensity can be accounted for by thermal fluctuations with a possible indication for some weak extra scattering. The assignment of the observed intensity to density fluctuations will be of importance for the interpretation of the dynamics observed in this low- $Q$ regime.

\section{B. Long-range density fluctuations}

The major and unexpected feature in the collective dynamics is the observation of a broad, weakly $Q$-dependent plateau of the collective relaxation times at $Q$ values smaller than those of the structure factor peak. Wavelength- 
dependent studies below and above the MS threshold have confirmed that the phenomenon is genuine. The temperature dependence of these times-and that is the second unexpected result - though observed at smaller momentum transfers, is weaker than that of the structural relaxation that shows itself at the structure factor maximum $Q_{\max }$.

As a consequence of the stronger temperature dependence at $Q_{\max }$, the slowing down effect in the peak region increases with decreasing temperature [see Figs. 9(a) and 11]. Furthermore, the apparent maximum of the relaxation times shifts from $Q \cong 0.8 \AA^{-1}$ at $390 \mathrm{~K}$ to $Q \cong 1 \AA^{-1}$ at $335 \mathrm{~K}$. At $Q \leqslant Q_{\max }$ the weaker temperature dependence leads to relaxation times that at high $T$ surpass even those of the structure factor maximum.

As we have shown in Sec. II, the low- $Q$ limit of the coherent scattering relates to the imaginary part of the susceptibility $\chi_{11}=1 / C_{11}$, where $C_{11}$ is the elastic modulus of the longitudinal sound waves. The elastic modulus $C_{11}$ relaxes with the stress relaxation time $\tau_{M}$. At $298 \mathrm{~K}$ from longitudinal sound wave damping in the $\mathrm{MHz}$ region $\tau_{M}=15.9 \mathrm{~ns}$ [24] is obtained. Extrapolating long-time stress-relaxation data [18] and dynamical shear data from lower frequency [20], $\tau_{M}=11.4 \mathrm{~ns} \tau_{M}=5.6 \mathrm{~ns}$ are obtained respectively. In view of the strong temperature dependence of $\tau_{M}$, the agreement is very reasonable.

In order to compare with the collective relaxation time $\tau_{\text {pair }}$ we need to take into account the relation between modulus and susceptibility relaxation time

$$
\tau_{\text {pair }}=\tau_{\chi}=\tau_{M} M(\omega \rightarrow \infty) / M(\omega \rightarrow 0),
$$

i.e., in order to obtain the susceptibility relaxation time one needs to multiply the modulus relaxation time by the factor $C_{11}(\omega \rightarrow \infty) / C_{11}(\omega \rightarrow 0)$. For polyisobutylene at $298 \mathrm{~K}$, $C_{11}(\omega \rightarrow \infty) \approx 8 \mathrm{GPa}$ and $C_{11}(\omega \rightarrow 0) \approx 2 \mathrm{GPa}$ [24]. From the longitudinal sound wave value one expects $\tau_{\text {pair }}=64 \mathrm{~ns}$ at $298 \mathrm{~K}$.

For higher temperatures, light scattering Brillouin data [23] show a loss maximum in $\tan \delta$ at $473 \mathrm{~K}$ for $4.95 \mathrm{GHz}$. The $\tan \delta$ is halfway between susceptibility and modulus, so the susceptibility relaxation time should be $\tau_{\text {pair }}=0.064 \mathrm{~ns}$. If we ascribe this behavior to an apparent activation energy we obtain a value of $E_{a}=0.48 \mathrm{eV}$.

While the low- $Q$ collective relaxation seems to follow a similar $T$ dependence as the stress relaxation, the structural relaxation, as observed at the structure factor maximum, clearly displays a stronger temperature dependence.

In Fig. 9(a) we display the interpolated mechanical susceptibility relaxation times as arrows. As may be seen, they nearly quantitatively agree with the pair relaxation times in the low- $Q$ regime, giving further support to the anomalous temperature dependence found in this regime. We emphasize once again that the short-range order displaying itself by the structure factor peak decays with a different temperature law.

\section{Self-motion}

In Gaussian approximation the self-correlation function assumes the form
TABLE I. Temperature dependence of the prefactor of the characteristic time for the self-motion, the structural relaxation time, and the mean squared displacement of the protons at this time.

\begin{tabular}{cccc}
\hline \hline$T(\mathrm{~K})$ & $a_{\text {self }}\left(\mathrm{ns} \AA^{2 / 0.55}\right)$ & $\tau_{\text {st }}(\mathrm{ns})$ & $\left\langle r^{2}\left(\tau_{\mathrm{st}}\right)\right\rangle\left(\AA^{2}\right)$ \\
\hline 335 & 1.23 & 23 & 30 \\
365 & 0.33 & 4 & 24 \\
390 & 0.15 & 1 & 17 \\
\hline \hline
\end{tabular}

$$
S_{\text {self }}(Q, t)=\exp \left[-\frac{Q^{2}}{6}\left\langle r^{2}(t)\right\rangle\right]
$$

where $\left\langle r^{2}(t)\right\rangle$ is the mean squared displacement of the scattering centers, in our case the protons. As it has been shown recently [42], for a homogeneous ensemble, where during the observation time each proton exhibits the same average fate, the combination of Eq. (31) and Eq. (24) leads to the power law dependence of the characteristic time given by Eq. (26). As may be seen from Fig. 4, the incoherent relaxation times, taken at different $Q$ and temperatures, follow this criterion well. Comparing the time scale with that of the structural relaxation measured at the structure factor peak, our data manifest that for PIB a homogeneous scenario is assumed for times longer than about $\frac{1}{10} \tau_{\mathrm{st}}$. We note that this time threshold corresponds just to the spectroscopic time of the $\alpha$ process. Similar observations have been reported for molecular dynamics (MD) simulations on polyisoprene [43].

From Eq. (31) together with Eq. (26) the time-dependent proton mean squared displacement may directly read.

$$
\left\langle r^{2}(t)\right\rangle=\frac{6}{Q^{2}}\left(\frac{t}{a_{\text {self }} Q^{-2 / \beta}}\right)^{\beta}=6\left(\frac{t}{a_{\text {self }}}\right)^{\beta}\left(\AA^{2}\right) .
$$

For example, at $390 \mathrm{~K}$ within an observation time of $5.5 \mathrm{~ns}$ the proton mean square displacement rises to $\left\langle r^{2}(5.5 \mathrm{~ns})\right\rangle$ $=43.5 \AA^{2}$.

With the experimental result of different temperature dependences for the self-motion-it follows the same intermediate temperature law as the low- $Q$ collective dynamicsand the collective relaxation at $Q_{\max }$, Eq. (32) immediately implies that the mean square displacement a proton performs during the structural relaxation time must increase with decreasing temperature. Inserting $\tau_{\text {st }}$ into Eq. (32), Table I reveals the mean square displacements at $\tau_{\mathrm{st}}(T)$. We observe a significant increase in $\left\langle r^{2}\left(\tau_{\text {st }}\right)\right\rangle$ from $17 \AA^{2}$ at $390 \mathrm{~K}$ to about $30 \AA^{2}$ at $335 \mathrm{~K}$. Apparently, the average distance a proton has to move, in order to achieve the relaxation of the structure, is growing considerably with falling temperature. This feature needs closer scrutiny in the future.

The key for an understanding of the observed selfdynamics is its temperature dependence that we will now relate to earlier observations of intrachain viscosity effects. Our experiment revealed that the self-correlation function shifts with a weaker temperature dependence than the structural relaxation. A description in terms of an activated process leads to an activation energy of $E_{o}=0.43 \mathrm{eV}$, significantly lower than the apparent activation energy of the 
structural relaxation of $E_{\alpha}=0.67 \mathrm{eV}$. In this context it is interesting to note that NMR studies on the segmental dynamics in PIB melts reveal an activation energy of $E_{\mathrm{NMR}}$ $=0.46 \mathrm{eV}$ in a similar temperature range. Apparently on a local scale, segmental relaxation is possible through a number of pathways leading to an activation energy smaller than that of the structural relaxation. We note that these activation energies are close to that of the stress relaxation.

Studying the single chain dynamic structure factor $S_{\text {chain }}(Q, t)$ of a PIB melt a possible mechanism was recently uncovered [6]. While the low- $Q$ dynamics is well described in terms of the entropic Rouse relaxation, already at relatively small $Q$ values $\left(Q \geqslant 0.2 \AA^{-1}\right)$ significant deviations from the Rouse dynamics became evident. A careful analysis revealed that for $S_{\text {chain }}(Q, t)$ the intermediate $Q$ dynamics could be well described in terms of the intrachain viscosity model of Allegra and Ganazzoli [4,5]. This model assumes a local dissipative relaxation process that limits the entropic relaxation towards shorter length scales. Such a process could be composed, e.g., of combined jump processes over rotational barriers and would be behind an internal viscosity force arising from local departures from configurational equilibrium. We note in passing that in PIB solutions the corresponding relaxational process could recently be identified directly as a jump over a single rotational barrier [45].

In the model the dissipative process is described by a Debye process with a single relaxation time $\tau_{A}$. This local mode couples to the Rouse modes, hybridizes with them and distorts the Rouse spectrum such as to explain quantitatively the observed single chain dynamic structure factor in PIB. In the evaluation it was found that the coupling effect was at full strength for Rouse mode numbers around 10. For the then investigated chain of 69 monomers this defines a length scale of about seven monomers. With the characteristic ratio of PIB $C_{\infty}=6.73$ and a $C$ - $C$ bond length of $\ell_{o}=1.54 \AA$, seven monomers span an end-to-end distance of $\sqrt{\left\langle R_{e}^{2}\right\rangle}$ $=\sqrt{\ell_{0}^{2} C_{\infty} 2 N}=15 \AA$. On this length scale the dissipative process ought to dominate the single chain structure factor. The analysis led to a temperature dependence with an activation energy of $E_{a}^{\tau_{A}}=0.43 \pm 0.1 \mathrm{eV}$ and a characteristic time $\tau_{A}=2.5+0.8,-0.3 \mathrm{~ns}$ at $390 \mathrm{~K}$, close to the values $0.7-1.5$ ns observed in the low- $Q$ collective relaxation and the interpolated value $0.8 \mathrm{~ns}$ of the stress relaxation time in $1 / C_{11}$.

With the parameters obtained from the analysis of $S_{\text {chain }}(Q, t)$ we calculated the model prediction for the selfcorrelation function. Figure 12 compares the extracted average relaxation times with those from our incoherent measurements at $390 \mathrm{~K}$. In the $Q$ range of applicability nearly quantitative agreement is obtained.

Thus, we have established a relationship between the observed self-correlation function and the intrachain viscosity effect present in the single chain structure factor. Using Eq. (32) we may even evaluate the mean square proton displacement related to this, the Rouse relaxation limiting process in PIB. Inserting $\tau_{A}$ into Eq. (32) we obtain $\left\langle r^{2}\left(\tau_{A}\right)\right\rangle$ $=27.7 \AA^{2}, 84 \%$ of the interchain distance of $d_{\text {chain }}=6.3 \AA$.

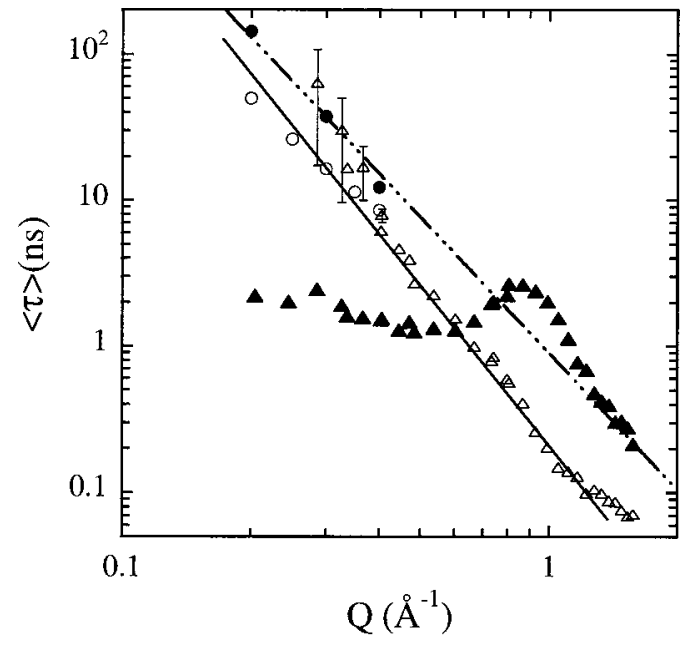

FIG. 12. Momentum transfer dependence of the average times at $390 \mathrm{~K}$ corresponding to: the self-correlation function $(\triangle)$, the collective motion $(\boldsymbol{\Delta})$, and the single chain structure factor $(\boldsymbol{O})$. The deduced values for the self-correlation obtained from the description of the single chain dynamic structure factor in terms of the model proposed by Allegra and Ganazzoli are also shown $(\bigcirc)$. The solid line represents a $Q^{-2 / 0.55}$ power law, and the dashed-dotted the extrapolation of the single chain structure factor times to higher $Q$-values.

\section{Collective intermediate-scale dynamics}

\section{1. de Gennes narrowing}

We may try to connect collective and self-dynamics through the commonly used picture of de Gennes narrowing [7]. However, such a scenario breaks down early on approaching lower $Q$ values. We demonstrate this behavior using Sköld's formulation [9],

$$
\widetilde{S}_{\text {pair }}(Q, t) \approx \widetilde{S}_{\text {self }}\left(\frac{Q}{\sqrt{S(Q)}}, t\right) .
$$

Assuming for $\widetilde{S}_{\text {pair }}(Q, t)$ and $\widetilde{S}_{\text {self }}(Q, t)$ the form of a stretched exponential [Eq. (24)], and taking into account the $Q$ dependence of $\tau_{\text {self }}$ given by Eq. (26), Eq. (33) implies

$$
\tau_{\text {pair }}(Q, T)=a_{\text {self }}(T)\left(\frac{Q}{\sqrt{S(Q)}}\right)^{-2 / \beta} .
$$

Figure 11 displays the result in comparing the prediction of Eq. (34) with the actually observed collective times. Obviously this approach fails not only quantitatively but also qualitatively. Neither the strong increase of the collective times relative to the self-motion in the peak region of $S(Q)$ is explained - this is the quantitative failure-nor the low- $Q$ plateau of $\tau_{\text {pair }}(Q)$ is predicted - this is the qualitative shortcoming. We note, however, that the self-motion times reflecting the proton motion may come out somewhat shorter than the times related to the pair correlation function that measures the displacements of all atoms (hydrogens and carbons). To take account of this effect we arbitrarily shifted the Sköld prediction by a factor of 2.5 such that it describes 
optimally the structure factor regime (dashed-dotted line in Fig. 11). Then the low- $Q$ profile of $\tau_{\text {pair }}(Q)$ is well described up to the plateau regime.

\section{Mode coupling theory}

The details of the derivation and predictions of mode coupling theory (MCT) for the structural relaxation of glassy liquids can be found in $[1,2]$. For the $Q$-dependence of the characteristic time of the $\alpha$ relaxation, MCT predicts,

$$
\tau_{\text {pair }} \propto\left[\frac{f_{Q}}{h_{Q}}\right]^{(2 / b)},
$$

where $f_{Q}$ and $h_{Q}$ are the relaxation strengths of the $\alpha$ and $\beta$ (fast) relaxations, respectively, and $b$ is the von Schweidler exponent.

From our analysis of the experimental data in terms of KWW functions, the values of the strengths $f_{Q}$ and $h_{Q}$ are known: $f_{Q}=A_{\text {pair }}(Q)$, and $h_{Q}=1-A_{\text {pair }}(Q)$. The determination of the value for $b$ would require a thorough analysis of the density fluctuations in terms of the MCT, for which an extension of the experimental window towards faster times including the $\beta$ (fast) regime is demanded. This is certainly beyond the scope of this work. In a first approximation, we may assume $b=\beta$. MCT predicts universality of time scales. This prediction is incompatible with the observation of two different temperature dependences for the structural and the stress relaxation. Nevertheless, here we would like to explore the predicted $Q$ dependence. To do this, the ratio between the time scales at the different temperatures has been fixed to that observed for the viscosity [Eq. (27)]. In this way, the resulting $Q$ - and $T$ dependences predicted by MCT for the relaxation times have been obtained. They are represented in Fig. 9(a) (lines) in comparison with our experimental results (symbols). The agreement is quite good for the lowest temperature investigated $(335 \mathrm{~K})$. There, the modulation in the peak region as well as the modulation in the plateau are fairly reproduced by MCT predictions. However, the thermal evolution obtained from experiment and theory are clearly different. From Eq. (35) different apparent activation energies in the peak and in the low- $Q$ regime are obtained $-f_{Q}$ and $h_{Q}$ vary with temperature only in the low- $Q$-regime. However, the ratio between those apparent activation energies varies in the opposite direction compared to the experiment. This can be followed from direct inspection of Fig. 8. While around and above $Q_{\max } f_{Q}$ does not change with temperature, below the peak $f_{Q}$ decreases with increasing temperature. This translates into an additional decrease of the relaxation time with respect to its value at $Q_{\max }$. We can thus conclude that, though MCT predictions reproduce the $Q$ dependence at the lowest temperature investigated, it does not describe the thermal behavior of the collective response of PIB in the intermediate length scales.

\section{Connection to single chain dynamics}

Polymer chains are connected objects, where correlations at least within a given chain persist to $Q$ values far below the first structure factor peak and we ask whether there may be a possibility to directly observe the relaxation of these longerrange correlations in the $S(Q)$ plateau before the structure factor peak.

The importance of such single chain self-contributions has recently been emphasized in connection with the relaxation dynamics of polymer blends [46]. There it was shown that the self-concentration of a given chain within a control volume spanned by the Kuhn length $\ell_{K}=\ell_{0} C_{\infty}$ is detrimental for the dynamics of a blend. In this concept the selfconcentration within the control volume is given by

$$
\phi_{s}=\frac{\nu_{b} C_{\infty}}{\ell_{K}^{3}},
$$

where $\nu_{b}=M_{o} / k \rho_{M} N_{A}$ is the volume per main chain bond $\left(M_{o}\right.$, monomer molar volume; $k$, number of main chain bonds per monomer; $\rho_{M}$, mass density and $N_{A}$, Avogadro number). For PIB $\phi_{s}=0.31$, i.e., with a volume spanned around a given chain by $\ell_{K}$, this chain contributes with 0.31 of the volume.

Fortunately, we are able to answer the question about possible contributions of such direct correlations to the dynamics of $S_{\text {pair }}(Q, t)$ in the low- $Q$ regime experimentally. In our study of the single chain dynamic structure factor $S_{\text {chain }}(Q, t)$ we determined the corresponding relaxation times over a large- $Q$-regime. They are displayed in Fig. 12 and compared to the collective times. As may be seen, in the low- $Q$ regime the single chain relaxation times are up to nearly two orders of magnitude slower than $\tau_{\text {pair }}(Q)$. Obviously, the single chain relaxation is masked by the other chains and is invisible.

Finally, we note that in Fig. 12 the extrapolated single chain relaxation times coincide at high $Q$ beyond the structure factor maximum with the collective relaxations studied here. Apparently, there the scattering volumes are such that in both cases, that of a labeled single chain and that of a coherently scattering multichain system, the single chain dynamics is observed each time.

\section{SUMMARY AND CONCLUSIONS}

We have studied the intermediate scale dynamics of PIB investigating both self-motion and the collective motion by neutron spin echo spectroscopy. In particular, in the $Q$ range below the first structure factor peak, these are the first neutron results, to our knowledge, on the collective relaxations reaching far into the plateau regime of the static structure factor $S(Q)$.

$S(Q)$ itself was studied by triple axis spectroscopy in combination with SANS. Careful wavelength-dependent measurements through the multiple scattering threshold $2 k_{i}$ $=Q_{\max }$ established the amount of MS to about $30 \%$ of total low $Q$ scattering (worst case) and allowed a measurement of $S(Q \rightarrow 0)$ on an absolute scale. The result $S(Q \rightarrow 0) \approx 0.22$ is in quite good agreement with the thermodynamic prediction from density fluctuations of $S(Q \rightarrow 0)=0.183$ at room temperature. 
The collective dynamics was studied on $d$-PIB revealing results down to $Q=0.20 \AA^{-1}$ far below the first structure peak at $Q_{\max }=1 \AA^{-1}$. The salient results are as follows.

(i) Below $Q \approx 0.6 \AA^{-1}$ the collective relaxation times exhibit a plateaulike behavior with some tendency to increase towards the lowest $Q$.

(ii) The temperature dependence of these collective relaxation times follows the stress relaxation rather than the structural relaxation. It is in accordance with the weaker $T$ dependence of segmental motion seen by NMR or the AllegraGanazzoli intrachain dissipation process.

(iii) The structural relaxation time $\tau_{\mathrm{st}}$ as observed from the dynamic structure factor at the first peak shifts with the rheological shift factor. The absolute values of $\tau_{\mathrm{st}}$ are longer than the characteristic times for the low $Q$-density fluctuations or the relaxation time of the modulus. The discrepancy increases with decreasing temperature. Thus, we are confronted with a picture where the short-range order decays slower than the modulus.

(iv) Though the low- $Q$ plateau behavior of $\tau_{\text {pair }}$ resembles the predictions of MCT, the observed two time scales for stress and structural relaxation cannot be reconciled with MCT.

In the same range of $Q$ and $T$, as the collective response we also studied the self-correlation function investigating the incoherent scattering from protonated PIB. There we found the following:

(i) In the $Q$ regime of the low- $Q$ plateau of the collective response up to the structure factor maximum the selfcorrelation times follow a power law $\sim Q^{-2 / \beta}$. This power law signifies that in the observed time regime the dynamics is essentially Gaussian corroborating earlier findings [42].

(ii) The correlation time at $Q_{\max }$ is more than an order of magnitude faster than the structural relaxation time.

(iii) The temperature dependence equals that of the collective dynamics at low $Q$ and is significantly weaker than that of the structural time.

Collective motion and self-motion were studied in the same $Q-T$ range and may be directly related to each other. The following features evolve:

(i) With the incoherent relaxation measured over a wide $Q$ range the average mean squared proton displacement may be calculated explicitly for each temperature. Given the different temperature dependences of the self-correlation function and the structural relaxation, we find that the mean squared proton displacement $\left\langle r^{2}\left(\tau_{\text {st }}\right)\right\rangle$ takes place until the structural relaxation achieved increases from $17 \AA^{2}$ at $390 \mathrm{~K}$ to $30 \AA^{2}$ at $335 \mathrm{~K}$.

(ii) In terms of the de Gennes narrowing collective correlation and self-correlation should be related through the structure factor. This approach fails qualitatively in the low- $Q$ regime.

The experimental results on the self-motion could be successfully related to earlier single chain dynamic structure factor experiments. There it was found that the AllegraGanazzoli model of intrachain viscosity could successfully describe the limit of the global Rouse relaxation toward shorter length scales. The self-correlation function calculated on the basis of this model and the parameters obtained from the single chain structure factor quantitatively agree with the incoherent spectra obtained here and explain the observations in the $Q$ range of applicability.

In order to understand the low- $Q$ plateau in the collective response, we revisited the Mountain theory and rederived the coupling of the density fluctuations to local relaxation processes using explicitly the second spectral sum rule that is not fulfilled by Mountain's expression. The result was that the central line in the coherent spectrum should relax with the stress relaxation time. This expectation, found to be valid earlier for the hydrodynamic regime accessed by photon correlation spectroscopy, was now corroborated by the NSE experiment at much larger $Q$ values.

\section{ACKNOWLEDGMENTS}

J.C. and A.A. acknowledge support from the following projects: DGICYT, PB97-0638; GV, EX 1998-23; UPV/ EHU, 206.215-G20/98; 9/UPV 00206.215-13568/2001. Support from Donostia International Physics Center is also acknowledged by D.R., A.A., and J.C.

\section{APPENDIX: CALCULATION OF MODE MIXING OF SOUND WAVES AND LOCAL PROCESSES}

To calculate the amount of mode mixing, let us consider the elastic distortion at the center of the local mode,

$$
\varepsilon_{11}=\varepsilon_{0} \cos (\Omega t)=\varepsilon_{0} \operatorname{Re}\left(e^{i \Omega t}\right) .
$$

The equation of motion of the local mode is

$$
\ddot{A}_{\mathrm{loc}}+\gamma_{\mathrm{loc}} \dot{A}_{\mathrm{loc}}+\omega_{\mathrm{loc}}^{2} A=-\Lambda_{P} \varepsilon_{11},
$$

where $\omega_{\mathrm{loc}}$ is the frequency and $\gamma_{\mathrm{loc}}$ is the damping of the local mode. One gets forced oscillations of the local mode with the amplitude $A_{0}$ given by

$$
A_{0}=\frac{-\Lambda_{P} \varepsilon_{0}}{\omega_{\mathrm{loc}}^{2}-\Omega^{2}+i \gamma_{\mathrm{loc}} \Omega} .
$$

The average kinetic energy of the local mode is then

$$
E_{\text {kin, }, \text { loc }}=\frac{1}{4} \Omega^{2}\left|A_{0}\right|^{2}=\frac{1}{4} \frac{-\Lambda_{P}^{2} \varepsilon_{0}^{2} \Omega^{2}}{\left(\Omega^{2}-\omega_{\text {loc }}^{2}\right)^{2}+\gamma_{\text {loc }}^{2} \Omega^{2}} .
$$

This is to be compared to the average kinetic energy of the sound wave

$$
E_{\mathrm{kin}, \mathrm{loc}}=E_{\mathrm{pot}, \text { sound }}=\frac{1}{4} \rho \nu_{/}^{2} \varepsilon_{0}^{2} V=\frac{1}{4} N m \nu_{/}^{2} \varepsilon_{0}^{2},
$$

where $V$ is the sample volume and $N$ the number of atoms in the sample. From the ratio of the kinetic energies, one finds the fraction of the Brillouin phonon that participates in the local mode, 


$$
f_{\mathrm{loc}}=\frac{\Lambda_{P}^{2}}{N m \nu_{/}^{2}} \frac{\Omega^{2}}{\left(\Omega^{2}-\omega_{\mathrm{loc}}^{2}\right)^{2}+\gamma_{\mathrm{loc}}^{2} \Omega^{2}} \approx \frac{\Lambda_{P}^{2}}{N m \nu^{2} \Omega^{2}},
$$

where the latter approximation holds for both $\omega_{\text {loc }}$ and $\gamma_{\text {loc }}$ much below the Brillouin frequency $\Omega$. In the following, we assume this to be the case.

The total kinetic energy of the mixed mode is $k_{B} T / 2$. Since $f_{\text {loc }}$ is very small, one has a fraction $f_{\text {loc }}$ of this total kinetic energy in the local mode, and a fraction $1-f_{\text {loc }}$ in the original Brillouin phonon. Thus the Brillouin signal is reduced by $1-f_{\text {loc }}$. The validity of the second moment sum rule implies that the missing intensity should appear somewhere else. At these low $Q$, the one-phonon approximation is rather accurate, so the missing intensity should appear with the scattering function $F_{\text {loc }}$ of the localized mode,

$$
F_{\mathrm{loc}}(\omega)=\frac{2}{\pi} \frac{\gamma_{\mathrm{loc}}}{\left(\omega^{2}-\omega_{\mathrm{loc}}^{2}\right)^{2}+\gamma_{\mathrm{loc}}^{2} \omega^{2}}
$$

In the low-frequency range, this damped oscillator function approaches a Lorentzian with a half width at half maximum of $\omega_{\mathrm{loc}}^{2} / 2 \gamma_{\mathrm{loc}}$ for $\gamma_{\mathrm{loc}} \gg \omega_{\mathrm{loc}}$. However, unlike a Lorentzian, it has a finite zero and second moment,

$$
\int_{0}^{\infty} F_{\mathrm{loc}}(\omega) d \omega=\frac{1}{\omega_{\mathrm{loc}}^{2}} \text { and } \int_{0}^{\infty} F_{\mathrm{loc}}(\omega) \omega^{2} d \omega=1
$$

The validity of the second moment sum rule implies a $Q$-independent scattering function.
[1] See, e.g., W. Götze and L. Sjögren, Rep. Prog. Phys. 55, 241 (1992).

[2] See, e.g., W. Götze, in Liquids, Freezing and Glass Transition, edited by J. P. Hansen, D. Levesque, and J. Zinn-Justin, (North-Holland, Amsterdam, 1991).

[3] P. E. Rouse, Jr., J. Chem. Phys. 21, 1272 (1953).

[4] G. Allegra and F. Ganazzoli, Macromolecules 14, 1110 (1981).

[5] G. Allegra and F. Ganazzoli, in Advances in Chemical Physics, edited by I. Prigogine and S. A. Rice (Wiley, New York, 1989), Vol. 75 , p. 265.

[6] D. Richter, M. Monkenbusch, J. Allgaier, A. Arbe, J. Colmenero, B. Farago, Y. Cheol Bae, and R. Faust, J. Chem. Phys. 111, 6107 (1999).

[7] P. G. de Gennes, Physica (Amsterdam) 25, 825 (1959).

[8] G. Vineyard, Phys. Rev. 110, 999 (1959).

[9] K. Sköld, Phys. Rev. Lett. 19, 1023 (1967).

[10] H. R. Schober, C. Oligschleger, and B. B. Laird, J. Non-Cryst. Solids 156, 965 (1993).

[11] W. Kob, C. Donati, S. J. Plimpton, P. H. Poole, and S. C. Glotzer, Phys. Rev. Lett. 79, 2827 (1997).

[12] C. Bennemann, C. Donati, J. Baschnagel, and S. Glotzer, Nature (London) 399, 246 (1999).

[13] See, e.g., B. Frick and D. Richter, Science 267, 1931 (1995), and references therein.

[14] See, e.g., E. Donth, The Glass Transition. Relaxation Dynamics in Liquids and Disordered Materials (Springer, Berlin, 2001).

[15] See, e.g., F. Sette, M. H. Krisch, C. Masciovecchio, G. Ruocco, and G. Monaco, Science 280, 1550 (1998).

[16] E. R. Fitzgerald, L. D. Grandine, Jr., and J. D. Ferry, J. Appl. Phys. 24, 650 (1953); J. D. Ferry, L. D. Grandine, Jr., and E. R. Fitzgerald, ibid. 24, 911 (1953).

[17] W. Philippoff, J. Appl. Phys. 24, 685 (1953).

[18] A. V. Tobolsky and E. Catsiff, J. Polym. Sci. 19, 111 (1956).

[19] D. J. Plazek, M. N. Vranken, and J. W. Berge, Trans. Soc. Rheol. 2, 39 (1958).

[20] J. D. Ferry, Viscoelastic Properties of Polymers (Wiley, New York, 1970).

[21] P. J. Törmälä, J. Macromol. Sci. Rev. Macromol. Chem. C 17, 297 (1979).
[22] D. J. Plazek, X. D. Zheng, and K. L. Ngai, Macromolecules 25, 4920 (1992); K. L. Ngai, D. J. Plazek, and C. A. Bero, ibid. 26, 1065 (1993); D. J. Plazek, I.-C. Chay, K. L. Ngai, and C. M. Roland, ibid. 28, 6432 (1995).

[23] G. D. Patterson, J. Polym. Sci., Polym. Phys. Ed. 15, 455 (1977).

[24] R. S. Marvin, R. Aldrich, and H. S. Sack, J. Appl. Phys. 25, 1213 (1954).

[25] D. Richter, A. Arbe, J. Colmenero, M. Monkenbusch, B. Farago, and R. Faust, Macromolecules 31, 1133 (1998).

[26] B. Stoll, W. Pechhold, and S. Blasenbrey, Kolloid Z. Z. Polym. 250, 1111 (1972).

[27] N. G. McCrum, B. E. Read, and G. Williams, Anelastic and Dielectric Effects in Polymeric Solids (Wiley, London, 1967), p. 389.

[28] W. P. Slichter, J. Polym. Sci., Part C: Polym. Symp. 14, 33 (1966).

[29] R. Dejean de la Batie, F. Lauprêtre, and L. Monnerie, Macromolecules 22, 2617 (1989).

[30] A. Arbe, J. Colmenero, B. Frick, M. Monkenbusch, and D. Richter, Macromolecules 31, 4926 (1998).

[31] K. Karatasos, J. P. Ryckaert, R. Ricciardi, and D. Richter, Macromolecules 31, 4926 (1998).

[32] D. W. van Krevelen, Properties of Polymers (Elsevier, Amsterdam, 1976) $\left(C_{p}:\right.$ p. $84 ; \lambda_{\text {th }}:$ p. 527).

[33] J.-P. Hansen and I. R. McDonald, Theory of Simple Liquids, 2nd ed., (Academic Press, New York, 1986). Second moment sum rule: Chap. 7.4, Eq. (7.4.40), p. 220; Rayleigh-Brillouin scattering: Chap. 8.5, p. $275 \mathrm{ff}$.

[34] S. W. Lovesey, Theory of Neutron Scattering from Condensed Matter (Clarendon, Oxford, 1984).

[35] L. Reinhard, G. Fytas, E. W. Fischer, and L. Willner, Acta Polym. 47, 399 (1996).

[36] C. H. Wang and E. W. Fischer, J. Chem. Phys. 82, 632 (1985).

[37] M. Györ, Zs. Fodor, H.-C. Wang, and R. Faust, J. Macromol. Sci., Pure Appl. Chem. A31, 2053 (1994).

[38] B. Jacrot, Rep. Prog. Phys. 39, 911 (1976).

[39] See, e.g., F. Mezei, in Neutron Spin Echo, edited by F. Mezei, Lecture Notes in Physics (Springer-Verlag, Heidelberg, 1980), Vol. 28. 
[40] M. Russina, F. Mezei, R. Lechner, S. Longeville, and B. Urban, Phys. Rev. Lett. 84, 3630 (2000).

[41] J. Colmenero, A. Alegría, A. Arbe, and B. Frick, Phys. Rev. Lett. 69, 478 (1992).

[42] A. Arbe, J. Colmenero, M. Monkenbusch, and D. Richter, Phys. Rev. Lett. 81, 590 (1998).

[43] J. Colmenero, F. Alvarez, and A. Arbe, Phys. Rev. E 65, 041804 (2002).

[44] Strictly, in order to transfer to microscopic times the rheologi- cal shift factor needs to be divided by temperature. However, considering the steep temperature dependence of $a_{F}(T)$ and the narrow $T$ range investigated, this correction makes no difference and was omitted in this phenomenological discussion.

[45] A. Arbe, M. Monkenbusch, J. Stellbrink, D. Richter, B. Farago, K. Almdal, and R. Faust, Macromolecules 34, 1281 (2001).

[46] T. Lodge, T. P. Lodge, and T. C. B. Mcleish, Macromolecules 33, 5278 (2000). 